\title{
Ascending Combinatorial Auctions with Risk Averse Bidders
}

\author{
Kemal Guler ${ }^{1}$ - Martin Bichler ${ }^{2}$. \\ Ioannis Petrakis ${ }^{2}$
}

Published online: 29 September 2015

(C) Springer Science+Business Media Dordrecht 2015

\begin{abstract}
Ascending combinatorial auctions are being used in an increasing number of spectrum sales worldwide, as well as in other multi-item markets in procurement and logistics. Much research has focused on pricing and payment rules in such ascending auctions. However, recent game-theoretical research has shown that such auctions can even lead to inefficient perfect Bayesian equilibria with risk-neutral bidders. There is a fundamental free-rider problem without a simple solution, raising the question whether ascending combinatorial auctions can be expected to be efficient in the field. Risk aversion is arguably a significant driver of bidding behavior in high-stakes auctions. We analyze the impact of risk aversion on equilibrium bidding strategies and efficiency in a threshold problem with one global and several local bidders. Due to the underlying free-rider problem, the impact of risk-aversion on equilibrium bidding strategies of local bidders is not obvious. We characterize the necessary and sufficient conditions for the perfect Bayesian equilibria of the ascending auction mechanism to have the local bidders to drop at the reserve price. Interestingly, in spite of the free-riding opportunities of local bidders, risk-aversion reduces the scope of the non-bidding equilibrium. The results help explain the high efficiency of ascending combinatorial auctions observed in the lab.
\end{abstract}

\footnotetext{
$\bowtie$ Martin Bichler

bichler@in.tum.de

Kemal Guler

kemalgoler@bilkent.edu.tr

Ioannis Petrakis

petrakis.ioannis@mytum.de

1 Department of Industrial Engineering, Bilkent University, Ankara, Turkey

2 Department of Informatics (I18), TU München, Boltzmannstr. 3, Garching, 85748 Munich, Germany
} 
Keywords Combinatorial auction - Risk aversion · Bayesian-Nash equilibrium analysis

\section{JEL Classification D44}

\section{Introduction}

The need to buy or sell multiple objects arises in areas such as industrial procurement, logistics, or when governments allocate spectrum licenses or other assets. It is a fundamental topic and the question how multiple indivisible objects should be allocated via an auction has enjoyed renewed interest in recent years (Airiau and Sen 2003; Cramton et al. 2006; Day and Raghavan 2007; Xia et al. 2004). One of the key goals in this research literature is to develop mechanisms that achieve high (allocative) efficiency with a strong game-theoretical solution concept such as a dominant strategy or an ex-post Nash strategy, such that bidders have no incentive to misrepresent their valuations. In other words, the strategic complexity for bidders is low as they do not need prior information about other bidders' valuations. Allocative efficiency measures whether the auctioned objects finally end up with the bidders who value them the most, thus, representing a measure of social welfare.

Combinatorial auctions are the most general types of multi-object market mechanisms, as they allow selling or buying a set of heterogeneous items to or from multiple bidders (Cramton et al. 2006). Bidders can specify package (or bundle) bids, i.e., prices are defined for the subsets of items that are auctioned. The price is only valid for the entire bundle, and the bid is indivisible. For example, in a combinatorial auction a bidder might want to buy a bundle, consisting of item $x$ and item $y$, for a bundle price of $\$ 100$, which might be more than the sum of the item prices for $x$ and $y$, when sold individually. We will say that bidder valuations for both items are complementary in this case.

Many publications have focused on the computational complexity of the allocation problem in combinatorial auctions (Lehmann et al. 2006; Rothkopf et al. 1998). Computational complexity is often manageable in real-world applications with a low number of items and bidders. In terms of strategic complexity, the standard solution in mechanism design is the Vickrey-Clarke-Groves (VCG) mechanism, which achieves efficiency in dominant strategies, i.e., bidders cannot increase their payoff by deviating from a truthful revelation of their valuations (Clarke 1971; Groves 1973; Vickrey 1961).

The Vickrey-Clarke-Groves mechanism is actually the unique direct revelation mechanism with a dominant strategy equilibrium (Green and Laffont 1977), but it is rarely used in practice. One of the problems of the VCG mechanism is that the outcomes might not be in the core. This means that a set of losing bidders was willing to pay more than what the winners had to pay. Non-core outcomes lead to very low revenue for the auctioneer and possibilities for shill bidding (Ausubel and Milgrom 2006b). Also, in many markets bidders are simply reluctant to reveal their true valuations to an auctioneer in a single-round sealed-bid auction, and they prefer an ascending (multi- 
round) auction format which is more transparent and conveys information about the competition in the market.

Much recent research has therefore focused on ascending combinatorial auctions, i.e., generalizations of the single-item English auction where bidders can outbid each other iteratively (Drexl et al. 2009; Schneider et al. 2010; Xia et al. 2004). Such auctions are selecting a core-outcome wrt. the bids, because a coalition of losing bidders can always increase their bids to become winning as long as bidders still have a positive payoff.

Unfortunately, no ascending combinatorial auction format allows for a strong solution concept for general types of bidder valuations (Bikhchandani and Ostroy 2002). In other words, with sufficient prior information about other bidders' valuations, there could always be valuations where a bidder can profit from not bidding truthfully up to his valuation. Let's consider a simple market with two identical items and three bidders, which we refer to as the threshold model. One "global" bidder is only interested in the bundle of two items for a value of up to $\$ 100$, while each of the two "local" bidders wants only one of the items and has a value of $\$ 80$. Since the local bidders together are stronger than the global bidder (i.e., the sum of their one-item valuations is higher than the global bidder's two-item valuation), they could try to free-ride on each other by pretending to have lower valuations. For example, one of the local bidders could pretend to have a value of $\$ 21$ only and hope that the second local bidder outbids the global bidder together with his bid of \$21. Recently, Sano (2011) has analyzed a threshold model with two items and a simple button auction. They showed that with uncertainty about other local bidders' valuations, ascending combinatorial auctions can even lead to inefficient solutions, where a local bidder drops out too early such that the global bidder wins although this is not the efficient solution. The model requires a few simplifying assumptions to yield a closed-form perfect Bayesian equilibrium strategy, but it nicely captures the strategic challenges that bidders face.

In the threshold model with strong local bidders also the outcome of the VCG auction is not in the core and the winners pay less than what the losers could have paid (Goeree and Lien 2013). The total of the VCG payments in the above example is $\$ 40$, while the global bidder would have bid up to $\$ 100$. Only with strong restrictions on the bidder valuations such as if goods are substitutes, an ascending combinatorial auction can achieve a strong game-theoretical solution concept such as an ex-post equilibrium (Ausubel and Milgrom 2006a), where straightforward bidding maximizes bidders' utility independent of the valuations of other bidders such that there are no incentives for manipulation.

This can be considered a negative result, because combinatorial auctions are typically used when bidders do have complementary valuations, which casts doubts on the efficiency of the various combinatorial auction formats that have been developed in the recent years. Note that this result is not restricted to linear (item-level) prices or non-linear anonymous prices (Drexl et al. 2009; Schneider et al. 2010; Xia et al. 2004), but it is relevant for the entire literature on ascending multi-object auctions.

The result actually applies to any type of core-selecting combinatorial auction. For example, a two-stage core-selecting combinatorial clock auction (CCA) has been used in several countries world-wide recently to sell spectrum (Cramton 2013). In the CCA, bidder-optimal core-selecting payments are computed after an ascending 
and a sealed-bid auction phase. Cramton and Day (2012) developed a quadratic coreselecting payment rule, which minimizes the Euclidean distance from the Vickrey payments and which is nowadays in use in spectrum auctions around the world.

Overall, this suggests that ascending combinatorial auctions in the field might be prone to manipulation and inefficiency. For example, there are spectrum auctions in countries with many local operators bidding only on regional spectrum licenses and a few national bidders, who are interested in larger packages. These environments can be seen as straightforward extensions of the three-bidder threshold model and similar manipulation might well matter and lead to inefficiencies.

In contrast to the negative result by Sano (2011), lab experiments have consistently showed high efficiency of ascending combinatorial auctions when subjects had complementary valuations. Many of these experiments used simple threshold models similar to the one described above (Banks et al. 2003; Goeree and Holt 2010; Kwasnica et al. 2005; Scheffel et al. 2011). In this paper, we want to analyze if risk-aversion can explain high efficiency in ascending combinatorial auctions. As we will see, the impact of risk aversion on efficiency in this environment is not as obvious as in singleobject auctions. Modeling risk aversion is a non-trivial extension of the results in Sano (2011), but one that is central for understanding bidder behavior in such auctions in the field.

\subsection{Risk Aversion}

In high-stakes spectrum auctions it is unlikely that bidders are risk-neutral as the outcome of such an auction can impact the economic fate of a telecom substantially. So, smaller payoffs have a higher utility as long as bidders win, which can be modeled with a concave utility function. Risk aversion is an important phenomenon to be considered in auctions due to the uncertainties faced by bidders in auctions in general. It is a fundamental concept in expected utility theory (Arrow 1965; Pratt 1964) and widely used to explain overbidding behavior in first-price sealed-bid auctions (Chen and Plott 1998; Cox et al. 1988; Kirchkamp and Reiss 2011). Risk aversion leads to different revenue rankings of single-item auctions and is likely to have an ample effect on equilibrium strategies in core-selecting combinatorial auctions. In single-item first price sealed-bid auctions, Riley and Samuelson (1981) show that risk aversion leads to uniformly higher bids and thus higher revenue. Bidders increase their bids since they want insurance against the possibility of losing. Risk aversion was also analyzed in the context of all-pay single-item auctions (Fibich et al. 2006), and single-item auctions with infinitely many bidders (Fibich and Gavious 2010).

In core-selecting auctions, the experiments in Goeree and Lien (2009) show that local bidders do not drop out at a price of null in experiments, as theory would suggest, but they continue to bid further. One might expect that risk aversion serves as a natural explanation for this overbidding phenomenon also in core-selecting auctions. However, the equilibrium bidding strategies in single-item auctions cannot easily be extended to combinatorial auctions. While risk aversion does not affect the equilibrium strategy under the single-item English auction, it might well have an impact on the condition for a non-bidding equilibrium in an ascending core-selecting com- 
binatorial auction. The higher one local bidder bids, the higher is the probability of the other local bidders to drop out. Due to the non-increasing equilibrium bidding strategies, it is unclear if a risk-averse bidder should actually overbid in equilibrium and if risk-aversion can recover the efficiency losses and the low revenue observed by Goeree and Lien (2013) for sealed-bid and Sano (2011) for ascending core-selecting combinatorial auctions.

\subsection{Contributions}

While a large part of the literature on combinatorial auctions focuses on computational questions, we complement this literature with a game-theoretical analysis. The contributions of this paper are the following: We consider the environments that have been analyzed in the Bayesian literature on sealed-bid and ascending core-selecting combinatorial auctions by Sano (2011) but analyze the impact of risk aversion on equilibrium strategies, revenue, and efficiency. Understanding risk aversion is important for practical applications and the impact is not obvious as indicated above.

First, we characterize the necessary and sufficient conditions for a perfect Bayesian equilibrium of the ascending core-selecting auction mechanism to have the small bidders to drop at the reserve price. We do so for general environments with risk-averse bidders as well as arbitrary asymmetries across bidders with respect to initial wealth, value distributions, and risk attitudes. Our first result is a generalization of the condition for a non-bidding equilibrium in Sano (2011), which allows for arbitrary concave utility functions, reserve prices, and differences in initial wealth. This flexibility in all three parameters allows for the analysis of realistic scenarios.

Second, we provide comparative statics and show that risk aversion and bidder asymmetries affect the equilibrium outcomes in ways that can be systematically analyzed. The impact of risk aversion on equilibrium bidding strategies is not obvious, since bidding higher can allow the other local bidder to drop out. Unlike super modular games or games fulfilling the single-crossing property (Athey 2001), such as the first price single-item sealed-bid auction, the impact of risk aversion is not obvious in the ascending auction game. The game does not fulfill these properties, as we will show, and bidders cannot simply buy insurance against the possibility of losing by increasing their bids. Increasing bids may lead to a lower probability of winning. Theorem 3 is central and it indicates that even in this case, risk-aversion reduces the scope of the non-bidding equilibrium in the sense that dropping at the reserve price ceases to be an equilibrium as the bidders become more risk averse. We also analyze different wealth levels, asymmetries across local bidders, and stochastic dominance orderings of the distributions of valuations and their impact on non-bidding. Similar to Goeree and Lien (2009) and Sano (2011) we do not analyze the continuation game, when the condition for a non-bidding equilibrium does not hold. So far, there are no Bayesian models of ascending combinatorial auctions in general to our knowledge, and it is likely that no closed-form solutions of Bayesian equilibria exist. The analysis of the continuation game is a significant challenge beyond the focus of this paper. However, our results already shed light on the impact of risk aversion on efficiency. 
The remainder of the paper is structured as follows. Section 2 contains a formulation of the model with descriptions of the environment and the auction procedure, as well as some preliminary lemmas on the model elements. In Sect. 3, we characterize the necessary and sufficient conditions for an ascending core-selecting auction to have a perfect Bayesian equilibrium in which small bidders stop bidding at the reserve price. Section 4 discusses parametric cases with specific distribution and utility functions. Finally, Sect. 5 provides conclusions. Throughout the paper, proofs that are technical in nature are placed in the "Appendix".

\section{The Model}

We consider a stylized multiple-unit auction environment with two objects and three bidders. Two objects are to be sold to one or two of three potential buyers through a core-selecting auction. The two objects are indistinguishable from the bidders' valuation perspective. As in Goeree and Lien (2009) and Sano (2011) the environment has an a priori asymmetry in one of the bidders, referred to as the 'large bidder', demands two units of the object and has value zero for a single unit. The other bidders, referred to as 'small bidders', each demands a single unit and has zero valuation for the second unit.

We consider an independent private values (IPV) environment where each buyer has a private value for the object(s) which is unknown to the others. In addition to the asymmetry of small and large bidders with respect to the number of units demanded, we allow a full range of ex ante asymmetries across bidders with respect to the distribution of valuations, utility functions and initial wealth levels.

We first describe the elements of the bidding environment followed by the details of the auction mechanism.

\subsection{The Environment: Information, Valuations, and Utility Functions}

The auction environment with three bidders, $N=\{1,2,3\}$, is represented by the collection $e=\left\{\left(F_{1}, F_{2}, G\right),\left(u_{1}, u_{2}, u_{3}\right),\left(\omega_{1}, \omega_{2}, \omega_{3}\right)\right\}$, where the three tuples denote the valuation distributions, the utility functions, and the wealth levels, respectively, of the three bidders. We provide the details of the two parts of an environment next.

\subsubsection{Information and Valuations}

Each buyer has a private value for the object(s) which is unknown to the others. We denote the valuations by $V$ and index it by the bidders. Bidder $i$ 's valuation is a random variable $V_{i}$ with distribution function $F_{i}(\cdot)$ for $i=1,2$ or $G(\cdot)$ for $i=3$ which has a strictly positive and continuously differentiable density function $f_{i}(\cdot)$ or $g(\cdot)$ on its support $\left[\underline{v} i, \bar{v}_{i}\right]$. We set $\underline{v}_{1}=\underline{v}_{2}=\underline{v}_{3}=0, \bar{v}_{1}=\bar{v}_{2}=1, \bar{v}_{3}=2$. Bidders $i=1,2$ are only interested in a single unit, while bidder $i=3$ is only interested in the package of two units. Each bidder is interested in a single package only, and which package bidders are interested in is assumed to be public knowledge. All already referenced papers modeling core-selecting auctions as a Bayesian game are using the same environment, which can be considered the simplest scenario where the Vickrey 
auction is not in the core. In our paper, we want to understand what risk aversion does to core-selecting auctions in this environment, where we still have closed form solutions for risk-neutral bidders.

\subsubsection{Utility Functions and Risk Aversion}

Bidders are expected utility maximizers. Bidder $i$ has von-Neumann-Morgenstern utility function $u_{i}: \mathbb{R} \rightarrow \mathbb{R}$. If a bidder with value $v$ and current wealth $\omega$ wins and pays a price $b$, his utility is $u_{i}(v-b+\omega)$; his utility is $u_{i}(\omega)$ if he loses. We assume $u_{i}$ is twice continuously differentiable, with $u_{i}^{\prime}(x)>0$ and $u_{i}^{\prime \prime}(x)<0 \forall x$. Therefore, $u_{i}$ is concave and $i$ risk-averse. Since von-Neumann-Morgenstern utility functions are unique up to affine transformations, i.e., $u(x)$ and $a+c u(x)$ represent the same underlying preferences for any choice of real numbers $a$ and $c>0$, we normalize the utility functions so that $u(\underline{\omega})=0$ and $u(\bar{\omega})=1$ for some wealth levels $\underline{\omega}$ and $\bar{\omega}$ with $0 \leq \underline{\omega}<\bar{\omega}$.

We use the measure $A(x)=\frac{-u^{\prime \prime}(x)}{u^{\prime}(x)} \in \mathbb{R}^{+}$introduced by Pratt in his seminal work (Pratt 1964), to compare the risk aversion of different bidders. Bidder $i$ is more riskaverse than bidder $j$ iff $A_{i}$ (x) $>A_{j}$ (x) $\forall x$. For the main theorems and corollaries we do not assume a parametric form of the utility function. We will, however, discuss certain results also for CARA, DARA, CRRA utility functions, which we briefly introduce in a form also considering initial wealth of bidders. Also these utility functions are used for the analysis of parametric cases.

A bidder exhibits constant relative (to his wealth) risk aversion if his utility function is of the form ${ }^{1}$ :

$$
u(x ; \underline{\omega}, \bar{\omega}, \rho)=\frac{x^{1-\rho}-\underline{\omega}^{1-\rho}}{\bar{\omega}^{1-\rho}-\underline{\omega}^{1-\rho}} \quad \text { where } 0<\rho \neq 1,0 \leq \underline{\omega}<\bar{\omega} .
$$

The corresponding Arrow-Pratt measure is $A(x)=\frac{-u^{\prime \prime}(x)}{u^{\prime}(x)}=\frac{\rho x^{-\rho-1}}{x^{-\rho}}=\frac{\rho}{x}$.

For the case with $\rho=1$, taking the limit $\rho \rightarrow 1$ we obtain the logarithmic utility function $^{2} u(x ; \underline{\omega}, \bar{\omega}) n(x / \bar{\omega})$. CRRA is a case of the decreasing absolute risk aversion family (DARA), the Arrow-Pratt measure of which has the form $A(x)=\frac{1}{a x+b}, a>0$.

A bidder exhibits constant absolute (independent to his wealth) risk aversion if his utility function is of the form ${ }^{3}$ :

\footnotetext{
1 This representation follows from using the base utility function $\frac{x^{1-\rho}}{1-\rho}$ and selecting the parameters $a$ and $c$ in the representation $u(x)=c\left(\frac{x^{1-\rho}-a}{1-\rho}\right)$ so that $u(\underline{\omega})=0$ and $u(\bar{\omega})=1$. Specifically, $u(\underline{\omega})=0 \Rightarrow a=\underline{\omega}^{1-\rho}$, and $u(\bar{\omega})=c\left(\frac{\bar{\omega}^{1-\rho}-\underline{\omega}^{1-\rho}}{1-\rho}\right)=1 \Rightarrow c=\frac{1-\rho}{\bar{\omega}^{1-\rho}-\underline{\omega}^{1-\rho}}$.

2 This is obtained by using positive affine transformations of the base utility function $\ln (\mathrm{x})$, i.e., $\mathrm{u}(\mathrm{x})=$ $\mathrm{c} \ln (\mathrm{x})+\mathrm{a}$ with the normalization $\mathrm{u}(\underline{\omega})=0$ and $\mathrm{u}(\bar{\omega})=1$.

3 This representation is based on positive affine transformations of the base utility function $-e^{-\lambda x}$. Selecting the parameters $a$ and $c$ in the representation $u(x)=u(x)=c\left(a-e^{-\lambda x}\right)$ so that $u(\underline{\omega})=0$ and $u(\bar{\omega})=1$ yields the functional form we use. Specifically, $u(\underline{\omega})=0 \Rightarrow a=e^{-\lambda \underline{\omega}}$, and $u(\bar{\omega})=$ $c\left(e^{-\lambda \underline{\omega}}-e^{-\lambda \bar{\omega}}\right)=1 \Rightarrow c=\frac{1}{e^{-\lambda \underline{\omega}}-e^{-\lambda \bar{\omega}}}$.
} 


$$
u(x ; \underline{\omega}, \bar{\omega}, \lambda)=\frac{e^{-\lambda \underline{\omega}}-e^{-\lambda x}}{e^{-\lambda \underline{\omega}}-e^{-\lambda \bar{\omega}}} \quad \text { where } \lambda>0,0 \leq \underline{\omega}<\bar{\omega} \text {. Hence } A(x)=\lambda .
$$

\subsection{The Ascending Core-Selecting Auction}

We study a multi-unit clock auction with package bidding as in Goeree and Lien (2009) and Sano (2011), and follow the notation and exact rules of these papers. The economic environment is identical to Sano (2011), and we intentionally kept the notation and model description, because this allows for easier comparison of the results.

Again, before the auction starts, bidders decide whether they participate or not and make this decision public. In this ascending clock auction, a single clock visible to all bidders is used to indicate the per-unit price $p$ of the items. The clock starts at an initial price $p=r$ and increases continuously, such that the reserve price for the package is $2 r$.

Each bidder responds with the demand at the current prices. Bidders are restricted by the activity rule: they can never increase their demands. Demand is known, and we assume that small bidders can demand either one unit or zero and large bidders can demand either the package of two units or zero. For the specific environments where each bidder can demand 0 or $k$ units, bidder messages can take only two values and a bidder can indicate whether she is 'in' or 'out' (equivalently, 'continue' or 'stop') by pressing or releasing a button. In the case we consider, a bidder pressing the button at a price $p$ indicates his decision to stop bidding, that is, that his demand is zero at that price. Let $p_{i}$ be the price at which bidder $i$ drops from the auction.

The auctioneer raises the price until the allocation is determined on the basis of these reported values. As in Goeree and Lien (2009) and Sano (2011) we use the following tie-breaking rule: if two or more local bidders drop out at the same time, one is selected to drop out randomly, i.e., he has to leave the auction without winning, while the others are allowed to continue. The auction rules of this button auction and the known demand of each bidder describe a simple market, which allows for a formal analysis and closed form equilibrium strategies. Still these assumptions capture the strategic challenges that bidders face in the threshold model. Let us briefly revisit the possible outcomes of this auction as introduced by Sano (2011):

- Case 1 Bidder 3 first stops at $p_{3}$ such that bidders 1 and 2 each win and they both pay $p_{3}$.

- Case 2 Bidder 1 first stops at $p_{1}$ and bidder 3 stops next at $p_{3}>p_{1}$. Note that the efficient allocation has not yet been determined. The price continues to increase. If bidder 2 is active until $2 p_{3}-p_{1}$, then bidders 1 and 2 each win one unit, since the total value for small bidders is $p_{1}+p_{2}>2 p_{3}$. Bidder 1 pays $p_{1}$, and bidder 2 pays $2 p_{3}-p_{1}$.

- Case 3 Bidder 1 first stops at $p_{1}$ and bidder 3 stops next at $p_{3}>p_{1}$. If bidder 2 stops at $p_{2}<2 p_{3}-p_{1}$, bidder 3 wins both units. Since the total value for small bidders is $p_{1}+p_{2}<2 p_{3}$, bidder 3 pays the amount $p_{1}+p_{2}$ by the bidder-optimal core discounting.

- Case 4 Bidders 1 and 2 stop first and second at $p_{1}$ and $p_{2}$ respectively. Then, bidder 3 wins both units with price $p_{1}+p_{2}$ by bidder-optimal core discounting. 
Bidder-optimal core discounting is adopted in order to promote truthful bidding. This discounting affects the bidders' incentives. However, as we will see later, a considerable part of the analysis is, in fact, independent of the discounting. Indeed, in the model with two local and one global bidder, the combinatorial clock auction by Porter et al. (2003), the auction iBundle by Parkes and Ungar (2000), the Ascending Proxy Auction by Ausubel and Milgrom (2002), and the two-stage CCA (Cramton 2013) would all lead to the same result.

Sano (2011) shows that the large bidder has a weakly dominant strategy of truthful bidding independent of the history of other bidders' bids. He also shows that a small bidder has a weakly dominant strategy of truthful bidding after the other small bidder stops bidding. All bidders in his analysis are assumed to be risk neutral.

An interesting implication of the analysis in Sano (2011) is that the payment rule does not matter for the ascending mechanism we study. A winning small bidder 2 in case 2 would always drop out at the price $p_{2}$ where $p_{1}+p_{2}>2 p_{3}$. Bidder 2 would not have an incentive to increase his bid in the supplementary sealed-bid phase as it is used in the combinatorial clock auction (Bichler et al. 2013), and the VCG payment would be in the core with respect to the bids submitted. The same is true for the winners in all other cases, who would always pay what they bid.

\section{Non-bidding Equilibrium in Ascending Auctions}

In this section we derive the non-bidding equilibrium condition for general types of risk-averse utility functions and extend the analysis of risk-neutral bidders of Goeree and Lien (2009) and Sano (2011). Subsequently we describe the non-bidding equilibrium with respect to asymmetry in the small bidders' utility functions, wealth levels, and value distributions. Our initial analysis concerns an economy with two small and one large bidder but we will generalize the results to many bidders.

Throughout the paper, we use the term 'non-bidding equilibrium' to refer to a perfect Bayesian equilibrium in which the equilibrium bid $\beta_{i}\left(v_{i}, \varnothing\right)=\min \left\{v_{i}, r\right\}$ for each $i=1,2$ and all $v_{i} \in V=[0,1]$. The second argument refers to the bid history and $\beta_{i}\left(v_{i}, \emptyset\right)$ describes $\mathrm{s}$ the dropping price of $i$ under empty history, i.e., assuming no other bidder has dropped. Second, we will use the terms symmetry and asymmetry to refer to ex ante symmetry and asymmetry of small bidders. Thus, 'symmetric bidders' is used instead of more cumbersome 'ex ante symmetric small bidders' to refer to an environment where small bidders are identical ex ante with respect to valuation distributions, risk attitudes and wealth levels, i.e., when $F_{1}=F_{2}=F, u_{1}=u_{2}=u$, and $\omega_{1}=\omega_{2}=\omega$.

\subsection{The Threshold Model}

The following Theorem 1 is a generalization of Theorem 1 by Sano (2011) for bidders with arbitrarily risk-averse utility functions. This is an important but non-trivial extension as can be seen in the "Appendix". 
Theorem 1 In environments with ex ante symmetric small bidders the necessary and sufficient condition for existence of a non-bidding equilibrium is

$$
\int_{r}^{1} G(t+r)\left(\frac{f(t)}{1-F(r)}-L(u ; 1, t, r, \omega)\right) d t \geq 0 .
$$

For a utility function $u(z)$ the functional $L(u ; x, y, z)=\frac{u^{\prime}(x+z)}{u(y+z)-u(z)}$, for $0 \leq x \leq y$, plays a central role in the statement and derivation of our main result. Some properties of this functional, such as its relation to Arrow-Pratt measure of absolute risk aversion, may be of independent interest. We collect some observations on this functional before we state our results.

$$
L(u ; v, t, r, \omega)=\frac{u^{\prime}(v-t+\omega)}{u(v-r+\omega)-u(\omega)}
$$

For a utility function in CRRA family this functional takes the form

$$
L(u ; v, t, r, \omega)= \begin{cases}\frac{(1-\rho)(v-t+\omega)^{-\rho}}{(v-r+\omega)^{1-\rho}-\omega^{1-\rho}}=\frac{(1-\rho)\left(\frac{v-t}{\omega}+1\right)^{-\rho}}{\omega\left\{\left(\frac{v-r}{\omega}+1\right)^{1-\rho}-1\right\}} & \text { if } \rho \neq 1 \\ \frac{\ln (\underline{\omega} / \bar{\omega})}{(v-t+\omega) \ln ((v-r+\omega) / \omega)} & \text { if } \rho=1\end{cases}
$$

For the CARA family of utility functions, it becomes

$$
L(u ; v, t, r, \omega)=\frac{\lambda e^{-\lambda(v-t+\omega)}}{e^{-\lambda \omega}-e^{-\lambda(v-r+\omega)}}=\frac{\lambda e^{-\lambda(v-t)}}{1-e^{-\lambda(v-r)}}
$$

When small bidders are ex ante symmetric and risk-neutral, the necessary and sufficient condition for non-bidding equilibrium reduces to the condition in Theorem 1 of $\mathrm{Sano}^{4}$ :

$$
\int_{r}^{1} G(t+r)\left(\frac{f(t)}{1-F(r)}-\frac{1}{1-r}\right) d t \geq 0
$$

If we suppose zero reserve prices and risk neutrality, Eq. (2) reduces to $\int_{0}^{1} g(t)$ $(F(t)-t) d t \leq 0$, i.e., $F$ first-order stochastically dominates the uniform distribution. In environments with risk-aversion this is not a sufficient condition: Suppose $F, G$ are uniform distributions, $r=0$ and $u$ is a CRRA with $\rho=1, \omega=1$. The non-bidding equilibrium condition is violated [the integral in (1) evaluates to -0.029 whereas under risk-neutrality to 0]. This fact impacts positively on both efficiency and revenue of the package clock auction since the bidder who successfully stops at $r$, now reveals his valuation to a greater extent.

\footnotetext{
4 The condition in Sano is $\int_{r}^{1}(G(t+r)-G(2 r))\left(\frac{f(t)}{1-F(r)}-\frac{1}{1-r}\right) d t \geq 0$ but the term $-G(2 r)$ is redundant since $\int_{r}^{1} G(2 r)\left(\frac{f(t)}{1-F(r)}-\frac{1}{1-r}\right) d t=G(2 r)(1-1)=0$
} 
So far, we have assumed complete symmetry among the small bidders. In the following, we deviate from this assumption and discuss the impact of asymmetry with respect to the small bidders' utility function, wealth levels, and value distributions.

Corollary 1 In environments with asymmetric small bidders the necessary and sufficient condition for existence of a non-bidding equilibrium is

$$
\begin{aligned}
& \int_{r}^{1} G(t+r)\left(\frac{f_{2}(t)}{1-F_{2}(r)}-L\left(u_{1} ; 1, t, r, \omega_{1}\right)\right) d t \geq 0 \\
& \int_{r}^{1} G(t+r)\left(\frac{f_{1}(t)}{1-F_{1}(r)}-L\left(u_{2} ; 1, t, r, \omega_{2}\right)\right) d t \geq 0 .
\end{aligned}
$$

\subsection{Many Bidders Case}

In this section, we generalize the case with three bidders and analyze cases with $m$ small and $l$ large bidders. As in Theorem 3 in Sano (2011), large bidders retain the dominant strategy property. Also, the free-rider problem does not arise when there are many small bidders, and there is competition among small bidders. However, this generalization to risk-averse bidders yields substantial differences in the proof provided in the "Appendix".

Theorem 2 In environments with $m \geq 2$ ex ante symmetric small bidders and $l \geq 1$ ex ante symmetric large bidders, if

$$
\int_{s}^{1}(G(t+r)-G(2 s))^{l}\left(\frac{f(t)}{1-F(s)}-L(u ; 1, t, s, \omega)\right) d t \geq 0
$$

for each $l=1, \ldots, n$ and all $s \in[r, 1]$, then the following strategies constitute $a$ perfect Bayesian equilibrium:

- Each large bidder follows a truthful strategy.

- If more than two small bidders continue bidding, then each small bidder follows a truthful strategy.

- If only two small bidders continue bidding (along with a large bidders), then each small bidder stops immediately.

- If a bidder is the only active small bidder, then he follows a truthful strategy.

\subsection{Comparative Statics and Discussion of the Ascending Core-Selecting Auction}

We will now discuss the impact that different levels of risk aversion, different wealth levels, and stochastic orderings of valuation distributions have on the result. 


\subsubsection{Different Levels of Risk Aversion}

We compare two environments $e, \tilde{e}$ which differ only in the risk-aversion of small bidders, with $\tilde{e}$ being the less risk-averse one. The risk-aversion of the large bidder is dispensable in our analysis since he always has the dominant strategy of truthful bidding. Our main question is whether the increase of the risk-aversion of small bidders makes their non-bidding strategy less attractive. The ascending auction game does not fulfill the single crossing property (Athey 2001) and a bid increase does not lead to an increase of the probability of winning, as we will show with the next example. Therefore, the impact of risk-aversion cannot simply be explained as in the first-price single-item auction.

Suppose bidder 1 is a local bidder with a low valuation while bidder 2 is a local bidder with a high valuation. If bidder 1 increases his bid from $b$ to $b+\varepsilon$, i.e., stays longer in the auction, then the probability that bidder 2 becomes the first bidder to stop increases, since his bid may be in the interval $[b, b+\varepsilon]$. However, bidder 1 has a higher probability of winning if he is the first to stop and in this way forces bidder 2 with the high valuation to truthfully compete with the large bidder. If bidder 2 stops first, the probability that bidder 1 wins over the global bidder is lower, but there is a trade-off with bidder 2's payoff, if he is not allowed to drop out. Therefore, increasing the bid does not necessarily lead to an increase in the probability of winning. Also the single crossing property, which demands $\frac{\partial^{2} \pi(b, v)}{\partial b \partial v}>0$ where $\pi(b, v)$ is the expected payoff of a bidder with valuation $v$ and bid $b$, is violated since the derivative can be negative.

Despite these facts, Theorem 3 answers affirmatively our main question. There are situations where risk-averse bidders continue bidding whereas risk neutral (or less risk-averse) bidders do not. As a corollary, risk aversion has a positive impact on the efficiency and revenue of the auction with non-bidding at all being the worst case scenario.

Theorem 3 now analyzes the impact of risk aversion assuming arbitrary concave utility functions. It describes the main result of this paper. To compare risk-aversion the Arrow-Pratt measure is employed. Following Pratt (1964) bidder $i$ is considered as more risk-averse than $j$ iff the Arrow-Pratt measure of his utility function is greater or equal than $j$ 's for any wealth level.

Theorem 3 If the environment $e=\left\{F_{1}, F_{2}, G, u_{1}, u_{2}, u_{3}, \omega_{1}, \omega_{2}, \omega_{3}\right\}$ admits a nonbidding equilibrium then so does the environment $\tilde{e}=\left\{F_{1}, F_{2}, G, \tilde{u}_{1}, \tilde{u}_{2}, u_{3}, \omega_{1}, \omega_{2}\right.$, $\left.\omega_{3}\right\}$ where $\tilde{u}_{i}(x)$ is such that $\tilde{A}_{i}(x)<A_{i}(x)$, for $\forall x$ and $i=1,2$, i.e., where small bidders are less risk-averse.

In what follows, we provide some intuition about the result of Theorem 3 . We examine how the utilities change for the two strategies "drop at the reserve price $r$ " and "continue". In the risk-neutral case the profit of bidder 1 when dropping at $r$ is $\left(v_{1}-r\right)$ multiplied by the probability of winning when dropping at $r$. It is important to observe that this probability is independent of his valuation $v_{1}$ since bidder 1 bids the amount $r$ and whether he wins or not, depends solely on the other two bidders' bids, who behave truthfully, thus on their valuations. Additionally, this probability of winning 


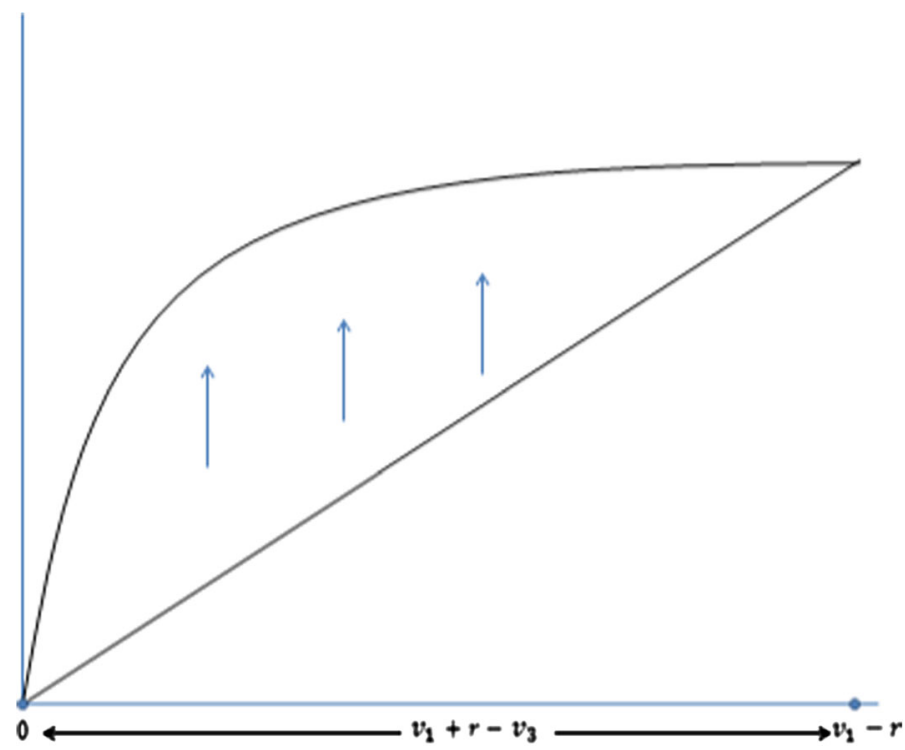

Fig. 1 Utility of wealth gained when winning by dropping at $r\left(\mathbf{v}_{\mathbf{1}}-\mathbf{r}\right)$ and continuing $\left(\mathbf{v}_{\mathbf{1}}+\mathbf{r}-\mathbf{v}_{\mathbf{3}}\right)$ for a risk-neutral and a concave utility function

is the same in both environments $e, \tilde{e}$. On the other hand, if bidder 1 decides to continue, his profit is $\left(v_{1}+r-v_{3}\right)$ times the probability of winning which is equal to the probability of the large bidder $v_{3}$ being in the interval $\left[2 r, v_{1}+r\right]$, since bidders 1 and 3 bid truthfully after bidder 2 has dropped at $r$. Also this probability is invariant in environments $e, \tilde{e}$. Since $v_{3} \in\left[2 r, v_{1}+r\right]$ when bidder 1 wins, his profit of continuing given that he wins is in the interval $\left[0, v_{1}-r\right]$ and is smaller than when dropping at $r$. The non-bidding equilibrium condition holds when the profits of dropping at $r$ are greater or equal than of continuing:

$$
\left(v_{1}-r\right) \operatorname{Prob}(\text { win } \mid \text { drop }) \geq\left(v_{1}+r-v_{3}\right) \operatorname{Prob}(\text { win } \mid \text { cont }) .
$$

We argued that changing the utility function from risk neutral to a strictly concave doesn't affect Prob (win $\mid$ drop), Prob (win $\mid$ cont) but only the utility of the quantities $\left(v_{1}-r\right)$, and $\left(v_{1}+r-v_{3}\right)$. Figure 1 shows how these change. For expositional purposes the two curves intersect at $\left(v_{1}-r\right)$.

The shape of the utility functions provides a rationale for our main result in this section. The utility when winning by dropping at $r$ is the same in both environments e, ẽ. On the contrary, the utility when winning by continuing at $r$ is higher in the risk-averse environment e for any monetary amount received. Therefore, the strategy of continuing becomes more attractive for risk-averse bidders and non-bidding ceases to be an equilibrium.

Corollary 2 Theorem 3 holds if $\tilde{u}_{1}(x)=a+b x, \tilde{u}_{2}(x)=a^{\prime}+b^{\prime} x\left(a, b, a^{\prime}, b, \in \mathbb{R}\right)$, and $u(x)$ is strictly concave for at least one $i=1,2\left(\right.$ otherwise $u_{i}(x)=\tilde{u}_{i}(x)$ ), i.e., 
in environment $\tilde{e}$ both local bidders are risk-neutral and in e at least one of them is risk averse.

Corollary 3 Theorem 3 holds if $u_{1}, u_{2}, \tilde{u}_{1}, \tilde{u}_{2}$ belong to the family of CARA utility functions and $\lambda_{1} \leq \tilde{\lambda}_{1}, \lambda_{2} \leq \tilde{\lambda}_{2}$.

Corollary 4 Theorem 3 holds if $u_{1}, u_{2}, \tilde{u}_{1}, \tilde{u}_{2}$ belong to the family of CRRA utility functions and $\rho_{1} \leq \tilde{\rho}_{1}, \rho_{2} \leq \tilde{\rho}_{2}$.

Corollary 5 The efficiency and revenue in environment e are greater than or equal to the efficiency and revenue in environment $\tilde{e}$.

Wealth levels and stochastic orderings of distributions can have ample effect on the results and are discussed in the following.

\subsubsection{Wealth Levels and Non-bidding Equilibrium}

We now examine the case where the initial wealth of one bidder increases by a positive amount $\delta$. CARA utility functions are independent of wealth. If bidders exhibit a CRRA or DARA utility function, then their risk aversion decreases whereas if they exhibit an IARA utility function, then risk aversion increases with higher wealth.

We will show that the left hand side of the non-bidding equilibrium (1) decreases if we add a positive amount of wealth $\delta$ as a corollary of Theorem 3 . The proof will follow the one of Theorem 3. However, we cannot apply the results of Theorem 3, because the utility function now remains the same and we cannot leverage $\tilde{A}_{i}(x)<A_{i}(x) \forall x$. Instead, we depart from the condition $A_{i}(\omega+\delta)<A_{i}(\omega), \delta>0$ (this is the case for CRRA, DARA).

Corollary 6 Suppose $u_{1}$ is a CRRA or DARA utility function. If the environment $e=\left\{F_{1}, F_{2}, F_{3}, u_{1}, u_{2}, u_{3}, \omega_{1}, \omega_{2}, \omega_{3}\right\}$ admits a non-bidding equilibrium then so does the environment $\tilde{e}=\left\{F_{1}, F_{2}, F_{3}, u_{1}, u_{2}, u_{3}, \tilde{\omega}_{1}, \omega_{2}, \omega_{3}\right\}$ where $\tilde{\omega}_{1}>\omega_{1}$.

\subsubsection{Stochastic Dominance Orderings and Non-bidding Equilibrium}

The conditions of first order stochastic dominance (FSD) and second order stochastic dominance (SSD) are:

$$
\begin{aligned}
& \tilde{H} \succcurlyeq_{F S D} H \Longleftrightarrow \tilde{H}(x) \leq H(x) \quad \forall x \text { (with strict inequality for some } \mathrm{x} \text { ) } \\
& \tilde{H} \succcurlyeq_{S S D} H \Longleftrightarrow \int_{0}^{x}(H(t)-\tilde{H}(t)) d t \geq 0 \quad \forall x \text { (with strict inequality for some } \mathrm{x} \text { ) }
\end{aligned}
$$

FSD implies SSD but not vice versa, hence FSD is a stronger condition. Variable $x$ first order stochastically dominates $y$ iff the probability that $x$ is higher than an amount $z$ is higher than the probability that $y$ is higher than this amount, for any $z$. Hence $x$ is stochastically larger than $y$ (it has a higher expected value). SSD mirrors the riskiness of two variables. If $x$ second order stochastically dominates $y$, then $x$ is less risky. Additionally, if the distributions of the random variables satisfy the single crossing property, then $x$ is also stochastically larger than $y$. 
We examine the impact of changing the distributions of the bidders' valuations to new ones that stochastically dominate the former ones. We show that changing the distribution of a valuation of a small bidder to a distribution which stochastically dominates the former leads to the non-bidding equilibrium being more probable. The reason is that the incentives to free ride increase in accordance with the probability that one small bidder outbids alone the large bidder.

Theorem 4 Suppose $r=0$. If the environmente $=\left\{F_{1}, F_{2}, G, u_{1}, u_{2}, u_{3}, \omega_{1}, \omega_{2}, \omega_{3}\right\}$ admits a non-bidding equilibrium then so does the environment $\tilde{e}=\left\{\tilde{F}_{1}, F_{2}, G, u_{1}\right.$, $\left.u_{2}, u_{3}, \omega_{1}, \omega_{2}, \omega_{3}\right\}$ where $\tilde{F}_{1} \succcurlyeq_{F S D} F_{1}$.

Theorem 5 Suppose additionally $g$ is non-increasing. Then the weaker condition $\tilde{F}_{1} \succcurlyeq_{S S D} F_{1}$ is sufficient for Theorem 4 to hold.

Theorems 4 and 5 hold for any reserve price $r$ if we impose first order stochastic dominance to the left-truncated versions of the cumulative distributions: $\tilde{F}_{1}(\cdot \mid r) \succcurlyeq_{F S D}$ $F_{1}(\cdot \mid r)$. Note that this condition is not implied by $\tilde{F}_{1} \succcurlyeq_{F S D} F_{1}$ since the stochastic dominance is not necessarily preserved after truncating.

\section{Analysis of Parametric Cases}

In what follows, we will analyze parametric cases of the sealed-bid and ascending core-selecting auctions.

\subsection{The Non-bidding Equilibrium Condition in the Ascending Auction}

We will illustrate selected parametric cases of the ascending auction assuming different types of distribution functions. Letting bidders be risk-neutral and all $v_{i}$ be drawn from a uniform distribution, (2) evaluates to 0 for all $r \in[0,1]$ leading to a non-bidding equilibrium. If $F$ is a truncated Gaussian distribution $F \sim N(0.5,0.25)$ on the interval $[0 \ldots 1]$ and $\mathrm{G} \sim N(1,0.5)$, then condition (2) is negative for $r \in[0 \ldots 1]$, and riskneutral bidders would continue bidding. Now if we increase the valuations of the small bidders and $F \sim N(0.8,0.4)$ then condition (2) is positive for most values of $r$ and the small bidders would try to drop (Fig. 2). The reason for free riding is that the probability for the competing small bidder to outbid the large bidder increases.

It is now interesting to understand, how risk aversion impacts this condition. If $v_{i}$ are uniformly distributed, the value of (1) is negative for both CARA and CRRA utility functions, and as opposed to the risk neutral case, the bidders would continue to bid (Fig. 3).

Figure 4 shows condition (2) for different values of $r$ when $F$ is a truncated Normal distribution $F \sim N(0.5,0.25)$ or $F \sim N(0.8,0.4)$ on the interval $[0 \ldots 1], G \sim$ $N(1,0.5)$ and we have a CARA utility function with $\lambda=0.9$. In Fig. 5 we change the utility function to a CRRA with $\rho=0.9, \omega=1$.

Last but not least, we vary the parameters $\lambda$ and $\rho$ and keep $r=0, F \sim N(0.8,0.4)$, $G \sim N(1,0.5)$ (Fig. 6). Condition (1) holds and lead to a non-bidding equilibrium 

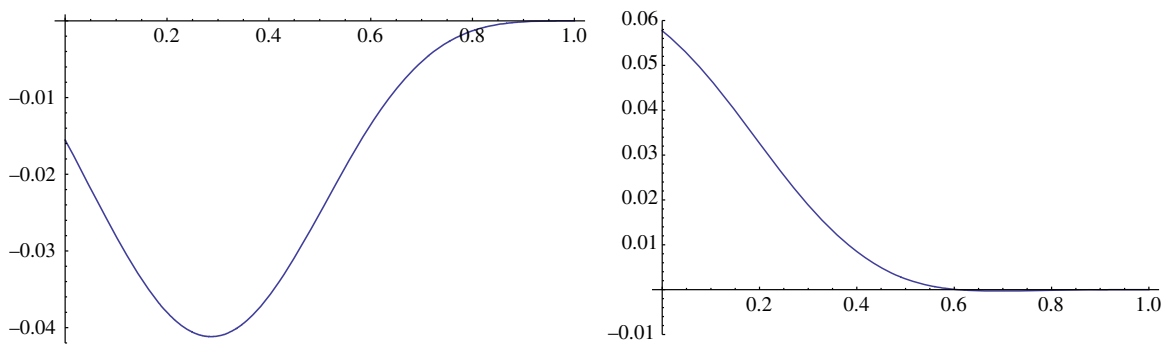

Fig. 2 Condition (2) for different values of $r$ with $\boldsymbol{F} \sim \boldsymbol{N}(\mathbf{0 . 5 , 0 . 2 5})$ on the left and $\boldsymbol{F} \sim \boldsymbol{N}(\mathbf{0 . 8 , 0 . 4})$ on the right with risk-neutral bidders
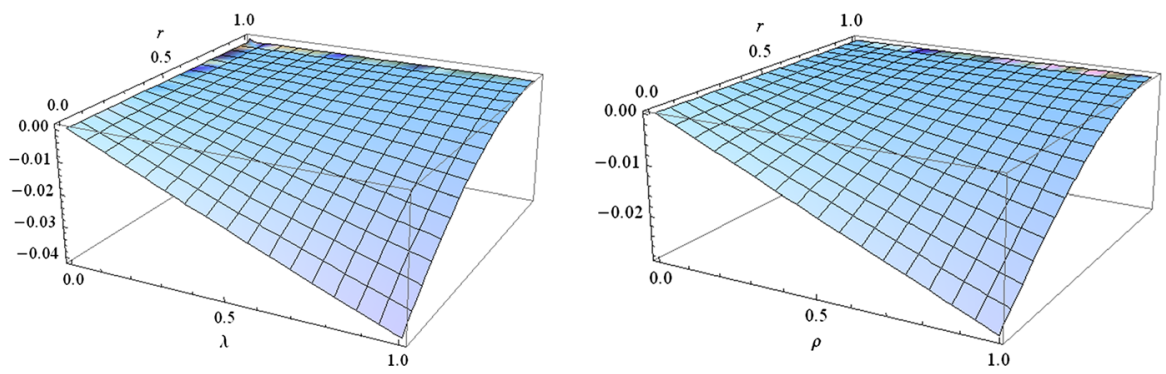

Fig. 3 Condition (1) for different values of $r, F \sim \boldsymbol{U}(\mathbf{0 , 1}), \boldsymbol{G} \sim \boldsymbol{U}(\mathbf{0 , 2})$. On the left for a CARA with $\lambda=0 \ldots 1$ and on the right CRRA utility function with $\rho=0 \ldots 1, \omega=1$
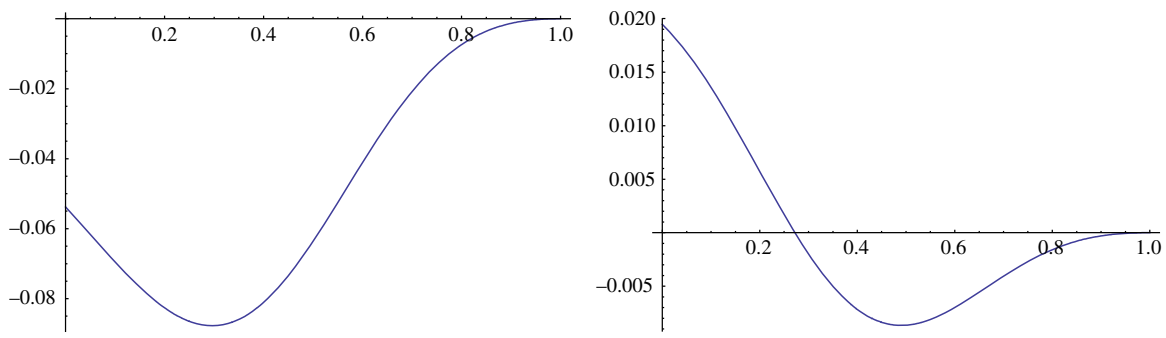

Fig. 4 Condition (2) for different values of $r$ with $\boldsymbol{F} \sim \boldsymbol{N}(\mathbf{0 . 5 , 0 . 2 5})$ on the left and $\boldsymbol{F} \sim \boldsymbol{N}(\mathbf{0 . 8 , 0 . 4})$ on the right with CARA utility functions
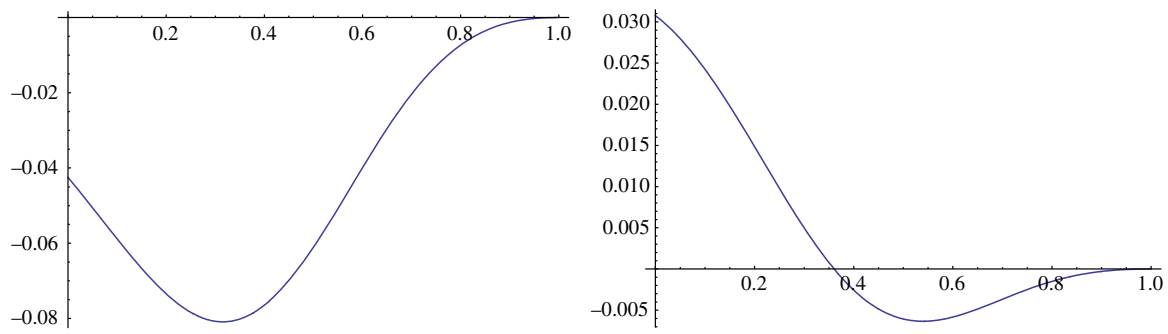

Fig. 5 Condition (1) for different values of $r$ with $\boldsymbol{F} \sim \boldsymbol{N}(\mathbf{0 . 5 , 0 . 2 5})$ on the left and $\boldsymbol{F} \sim \boldsymbol{N}(\mathbf{0 . 8 , 0 . 4})$ on the right with CRRA utility functions and $\rho=0.9, \omega=1$.) 

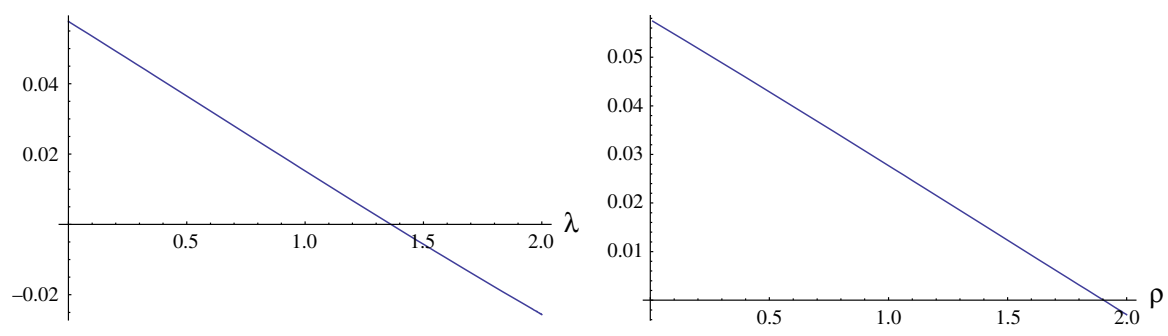

Fig. 6 Condition (1) with $\boldsymbol{F} \sim \boldsymbol{N}(\mathbf{0 . 8 , 0 . 4})$ for $r=0$ and different parameters of a CARA or CRRA utility function

for all values of $\rho$ and for $\lambda<1.4$. Higher values of $\lambda$ lead to such high risk aversion that the non-bidding equilibrium is eliminated.

\section{Conclusions}

Ascending combinatorial auctions have led to a fruitful stream of research on pricing rules in such auctions. The recent game-theoretical literature in this area casts doubts that such auctions are efficient in the field. The analysis of a simplified button auction in a threshold model rule shows that there can even be inefficient non-bidding equilibria (Goeree and Lien 2009; Sano 2011). Overall, the threshold model illustrates that there is potential for profitable manipulation and truthful bidding might not be in the interest of bidders. This model can easily be extended to real-world markets where local bidders compete against bidders interested in a global or national coverage as it is regularly the case in spectrum auctions. The game-theoretical results are in sharp contrast to the high allocative efficiency that combinatorial auctions achieved in lab experiments.

Previous game-theoretical work assumes risk-neutral bidders. Risk aversion is an important phenomenon in high-stakes auctions and it is important to understand its impact on equilibrium strategies, revenue and efficiency of these auctions. We stick to the environments and auction mechanisms, which have been analyzed so far, but take risk aversion into account. The free-rider problem among the two local bidders is such that the impact of risk aversion is not obvious, however. In this paper, we show that risk aversion, reserve prices, and bidder asymmetries affect the equilibrium outcomes in ways that can be systematically analyzed. Risk aversion reduces the scope of the non-bidding equilibrium in ascending core-selecting auctions.

Interesting questions remain open. First, the nature of equilibria when the nonbidding condition fails is perhaps the most important gap to be filled. Also, the analysis of other environments with more items or multi-minded bidders requires further analysis.

Acknowledgments The authors gratefully acknowledge funding from the German National Science Foundation (DFG BI-1057-7). Kemal Guler's work was undertaken during a visit to Bilkent University supported under a fellowship Grant from the TUBITAK BIDEP 2236 Co-Circulation Program. He gratefully acknowledges the financial support of TUBITAK and hospitality of Bilkent University. 


\section{Appendix}

Theorem 1 In environments with ex ante symmetric small bidders the necessary and sufficient condition for existence of a non-bidding equilibrium is

$$
\int_{r}^{1} G(t+r)\left(\frac{f(t)}{1-F(r)}-L(u ; 1, t, r, \omega)\right) d t \geq 0
$$

Proof of Theorem 1 Suppose bidder 2 selects to drop at $r$. For bidder 1, if he also selects to drop at $r$, his expected payoff is determined by the randomization that determines who gets to continue. If bidder 1 drops at $r$, and bidder 2 is selected to continue bidding in the tie-breaking lottery, bidder 1's expected utility is

$$
\begin{aligned}
\pi(d r o p ; v, r)= & u(v-r+\omega) \operatorname{Prob}\left\{1 \text { wins with bid } r \mid\left((r, v), \beta_{-1}\right)\right\} \\
& +u(\omega) \operatorname{Prob}\left\{1 \text { loses with bid } r \mid\left((r, v), \beta_{-1}\right)\right\} \\
= & u(\omega)+(u(v-r+\omega)-u(\omega)) \operatorname{Prob}\left\{1 \text { wins with bid } r \mid\left((r, v), \beta_{-1}\right)\right\} \\
= & u(\omega)+\frac{u(v-r+\omega)-u(\omega)}{1-G(2 r)}\left\{\int_{r}^{1} G(t+r) \frac{f_{2}(t)}{1-F_{2}(r)} d t-G(2 r)\right\}
\end{aligned}
$$

where we used the following derivations to evaluate the probability term,

$$
\begin{aligned}
& \operatorname{Prob}\left\{1 \text { wins with bid } r \mid\left((r, v), \beta_{-1}\right)\right\}=\operatorname{Prob}\left\{v_{2}+r>v_{3} \mid \bar{v}_{2} \geq v_{2} \geq r, v_{3} \geq 2 r\right\} \\
& =\frac{\operatorname{Prob}\left\{v_{2}+r>v_{3}, \bar{v}_{2} \geq v_{2} \geq r, v_{3} \geq 2 r\right\}}{\operatorname{Prob}\left\{\bar{v}_{2} \geq v_{2} \geq r, v_{3} \geq 2 r\right\}}=\frac{\operatorname{Prob}\left\{v_{2}+r>v_{3}, \bar{v}_{2} \geq v_{2} \geq r, v_{3} \geq 2 r\right\}}{\operatorname{Prob}\left\{\bar{v}_{2} \geq v_{2} \geq r\right\} \operatorname{Prob}\left\{v_{3} \geq 2 r\right\}} \\
& =\frac{\operatorname{Prob}\left\{v_{2}+r>v_{3} \geq 2 r, \bar{v}_{2} \geq v_{2} \geq r\right\}}{\left(1-F_{2}(r)\right)(1-G(2 r))}=\frac{\int_{r}^{\bar{v}_{2}} \int_{2 r}^{v_{2}+r} d G\left(v_{3}\right) d F_{2}\left(v_{2}\right)}{\left(1-F_{2}(r)\right)(1-G(2 r))} \\
& =\frac{\int_{r}^{\bar{v}_{2}}\left(G\left(v_{2}+r\right)-G(2 r)\right) d F_{2}\left(v_{2}\right)}{\left(1-F_{2}(r)\right)(1-G(2 r))}=\frac{\int_{r}^{\bar{v}_{2}} G(t+r) f_{2}(t) d t-G(2 r)\left(1-F_{2}(r)\right)}{\left(1-F_{2}(r)\right)(1-G(2 r))} \\
& =\frac{\int_{r}^{\bar{v}_{2}} G(t+r) \frac{f_{2}(t)}{1-F_{2}(r)} d t-G(2 r)}{1-G(2 r)}
\end{aligned}
$$

If bidder 1 does not drop at $r$, or if he drops but he is selected to continue in the tie-breaking lottery, his expected utility is

$$
\begin{aligned}
\pi(\text { continue } ; v), r)= & u(\omega)+\frac{u(v-r+\omega)-u(\omega)}{1-G(2 r)} \\
& \times\left\{\int_{r}^{v} \frac{u^{\prime}(v-y+\omega)}{u(v-r+\omega)-u(\omega)} G(y+r) d y-G(2 r)\right\}
\end{aligned}
$$

where we used the fact that 1 wins in the continuation game against bidder 3 in the event that $\left\{v+r>w_{3}\right\}$ and pays $w_{3}-r$ when he wins. 


$$
\begin{aligned}
\pi(\text { continue } ;, r) & =\frac{\int_{2 r}^{v+r} u(v+r-s+\omega) g(s) d s+\int_{v+r}^{\bar{w}_{3}} u(\omega) g(s) d s}{1-G(2 r)} \\
& =u(\omega)+\frac{\int_{2 r}^{v+r}(u(v+r-s+\omega)-u(\omega)) g(s) d s}{1-G(2 r)}
\end{aligned}
$$

Integration by parts, setting $y:=s-r$ and rearranging terms gives (6).

If the ties are broken via a lottery that selects bidder 1 with probability $q$ and bidder 2 with probability $1-q$, expected utility of dropping at $r$ for bidder 1 is

$$
\begin{aligned}
E U(\text { drop } ; v, r) & =q \pi(\text { drop } ; v, r)+(1-q) \pi(\text { continue } ; v, r) \\
& =\pi(\text { continue } ; v, r)+q(\pi(\text { drop } ; v, r)-\pi(\text { continue } ; v, r))
\end{aligned}
$$

Therefore, the expected utility difference between the two actions for bidder 1 is

$$
\begin{aligned}
\Delta E U(v, r):= & E U(\text { drop; } v, r)-E U(\text { continue } ; v, r) \\
= & q(\pi(d r o p ; v, r)-\pi(\text { continue } ;, r)) \\
= & q\left(\frac{u(v-r+\omega)-u(\omega)}{1-G(2 r)}\left\{\int_{r}^{1} G(t+r) \frac{f_{2}(t)}{1-F_{2}(r)} d t-G(2 r)\right\}\right. \\
& -\frac{u(v-r+\omega)-u(\omega)}{1-G(2 r)} \\
& \left.\times\left\{\int_{r}^{v} \frac{u^{\prime}(v-y+\omega)}{u(v-r+\omega)-u(\omega)} G(y+r) d y-G(2 r)\right\}\right) \\
= & q \frac{u(v-r+\omega)-u(\omega)}{1-G(2 r)}\left\{\int_{r}^{1} G(t+r) \frac{f_{2}(t)}{1-F_{2}(r)} d t\right. \\
& \left.-\int_{r}^{v} \frac{u^{\prime}(v-y+\omega)}{u(v-r+\omega)-u(\omega)} G(y+r) d y\right\}
\end{aligned}
$$

Remark Note that the sign of the expected utility difference is independent of the value of $q$.

$\operatorname{sign} \Delta E U(v, r)=\operatorname{sign}\left\{\int_{r}^{1} G(t+r) \frac{f_{2}(t)}{1-F_{2}(r)} d t-\int_{r}^{v} L(v, t, r, \omega) G(t+r) d t\right\}$

Thus the condition that bidder 1's expected utility from dropping at $r$ is at least as high as his expected utility from continuing becomes

$$
\begin{aligned}
\Delta E U(v, r)>0 \quad \forall v & \Longleftrightarrow \int_{r}^{1} G(t+r) \frac{f_{2}(t)}{1-F_{2}(r)} d t \\
& >\int_{r}^{v} L(v, t, r, \omega) G(t+r) d t \forall v
\end{aligned}
$$




$$
\begin{aligned}
& \Longleftrightarrow \int_{r}^{1} G(t+r) \frac{f_{2}(t)}{1-F_{2}(r)} d t \\
& >\max _{v} \int_{r}^{v} L(v, t, r, \omega) G(t+r) d t
\end{aligned}
$$

We show in Lemma 1 that $T(v):=\int_{r}^{v} L(v, t, r, \omega) G(t+r) d t$ is monotone increasing in $v$ and thus it is maximized when $v=1$ with maximum value that is equal to $\int_{r}^{1} L(1, t, r, \omega) G(t+r) d t$

Therefore,

$$
\begin{aligned}
\Delta E U(v, r)>0 \forall v & \Longleftrightarrow \int_{r}^{1} G(t+r) \frac{f_{2}(t)}{1-F_{2}(r)} d t>\int_{r}^{1} L(1, t, r, \omega) G(t+r) d t \\
& \Longleftrightarrow \int_{r}^{1} G(t+r)\left(\frac{f_{2}(t)}{1-F_{2}(r)}-L(1, t, r, \omega)\right) d t>0
\end{aligned}
$$

Lemma $1 T(v):=\int_{r}^{v} L(v, t, r, \omega) G(t+r) d t$ is monotone increasing in $v$.

Proof Lemma 1 First we prove some claims:

\section{Claim 1}

$$
\begin{gathered}
\text { (a) } \frac{\partial L(v, t, r, \omega)}{\partial v}=-\frac{\partial L(v, t, r, \omega)}{\partial t}-L(v, t, r, \omega) L(v, r, r, \omega) \\
=\frac{u^{\prime \prime}(v-t+\omega)}{u(v-r+\omega)-u(\omega)}-L(v, t, r, \omega) L(v, r, r, \omega) \\
\text { (b) } L(v, r, r, \omega)-L(v, v, r, \omega)=\frac{u^{\prime}(v-r+\omega)-u^{\prime}(\omega)}{u(v-r+\omega)-u(\omega)}
\end{gathered}
$$

Proof of Claim 1 Directly from $L(v, t, r, \omega)$ definition.

\section{Claim 2}

$$
\int_{r}^{v} \frac{u^{\prime \prime}(v-t+\omega)}{u(v-r+\omega)-u(\omega)} G(t+r) d t>G(v+r) \frac{u^{\prime}(v-r+\omega)-u^{\prime}(\omega)}{u(v-r+\omega)-u(\omega)}
$$

\section{Proof of Claim 2}

$$
\begin{aligned}
& G(t+r)<G(v+r) \Longleftrightarrow \frac{u^{\prime \prime}(v-t+\omega)}{u(v-r+\omega)-u(\omega)} G(t+r) \\
& >\frac{u^{\prime \prime}(v-t+\omega)}{u(v-r+\omega)-u(\omega)} G(v+r) \\
& \Longleftrightarrow \int_{r}^{v} \frac{u^{\prime \prime}(v-t+\omega)}{u(v-r+\omega)-u(\omega)} G(t+r) d t
\end{aligned}
$$




$$
\begin{aligned}
& >G(v+r) \int_{r}^{v} \frac{u^{\prime \prime}(v-t+\omega)}{u(v-r+\omega)-u(\omega)} d t \\
& \Longleftrightarrow \int_{r}^{v} \frac{u^{\prime \prime}(v-t+\omega)}{u(v-r+\omega)-u(\omega)} G(t+r) d t>G(v+r) \frac{u^{\prime}(v-r+\omega)-u^{\prime}(\omega)}{u(v-r+\omega)-u(\omega)}
\end{aligned}
$$

Claim $3 T(v)<G(v+r)$

Proof of Claim 3 Using the fact that $G(x)$ is increasing, we get

$$
\begin{aligned}
T(v) & =\int_{r}^{v} L(v, t, r, \omega) G(t+r) d t=\frac{\int_{r}^{v} u^{\prime}(v-t+\omega) G(t+r) d t}{u(v-r+\omega)-u(\omega)} \\
& <\frac{G(v+r) \int_{r}^{v} u^{\prime}(v-t+\omega) d t}{u(v-r+\omega)-u(\omega)}=G(v+r)
\end{aligned}
$$

Now we prove Lemma 1 by showing $T^{\prime}(v)>0$

$$
\begin{aligned}
T^{\prime}(v)= & L(v, v, r, \omega) G(v+r)+\int_{r}^{v} \frac{\partial L(v, t, r, \omega)}{\partial v} G(t+r) d t \\
= & L(v, v, r, \omega) G(v+r)-\int_{r}^{v} \frac{-u^{\prime \prime}(v-t+\omega)}{u(v-r+\omega)-u(\omega)} G(t+r) d t+ \\
& -L(v, r, r, \omega) \int_{r}^{v} L(v, t, r, \omega) G(t+r) d t \quad \text { (Claim 1a) } \\
= & L(v, v, r, \omega) G(v+r)+\int_{r}^{v} \frac{u^{\prime \prime}(v-t+\omega)}{u(v-r+\omega)-u(\omega)} G(t+r) d t \\
& -L(v, r, r, \omega) T(v)(T(v) \operatorname{definition)} \\
> & L(v, v, r, \omega) G(v+r)+G(v+r) \frac{u^{\prime}(v-r+\omega)-u^{\prime}(\omega)}{u(v-r+\omega)-u(\omega)} \\
& -L(v, r, r, \omega) T(v) \quad(\operatorname{Claim} 2) \\
= & L(v, v, r, \omega) G(v+r)+G(v+r)(L(v, r, r, \omega)-L(v, v, r, \omega)) \\
& -L(v, r, r, \omega) T(v) \quad(\text { Claim 1b) } \\
= & L(v, r, r, \omega)(G(v+r)-T(v))>0 \quad(\text { Claim } 3 \text { and } u(x) \text { concave) }
\end{aligned}
$$

Theorem 2 In environments with $m$ ex ante symmetric small bidders and $l$ ex ante symmetric large bidders, if

$$
\int_{s}^{1}(G(t+r)-G(2 s))^{l}\left(\frac{f(t)}{1-F(s)}-L(u ; 1, t, s, \omega)\right) d t \geq 0
$$

for each $l=1, \ldots, n$ and all $s \in[r, 1]$, then the following strategies constitute $a$ perfect Bayesian equilibrium: 
1. Each large bidder follows a truthful strategy.

2. If more than two small bidders continue bidding, then each small bidder follows a truthful strategy.

3. If only two small bidders continue bidding (along with a large bidders), then each small bidder stops immediately.

4. If a bidder is the only active small bidder, then he follows a truthful strategy.

Proof of Theorem 2 Each large bidder follows obviously a truthful strategy. If more than two small bidders continue bidding, each of them follows a truthful strategy (otherwise who stops loses immediately). Hence, it only needs to be shown that if two small bidders continue bidding (along with a large bidder), then each small bidder stops immediately.

Let $s$ be the current price level.

$\mathrm{H}_{1}(\cdot \mid \cdot)$ denotes the conditional CDF of $\mathrm{w}^{(1)}$ among 1 valuations and $\mathrm{h}_{1}(\cdot \mid \cdot)$ the corresponding pdf

$$
\begin{aligned}
\text { e.g., } H_{l}\left(v_{2}+s \mid w^{(l)} \geq 2 s\right) & =\frac{\left(G\left(v_{2}+s\right)-G(2 s)\right)^{l}}{(1-G(2 s))^{l}} \text { and } h_{l}\left(v_{2}+s \mid w^{(l)} \geq 2 s\right) \\
& =\frac{g\left(v_{2}+s\right)\left(G\left(v_{2}+s\right)-G(2 s)\right)^{l-1}}{(1-G(2 s))^{l}}
\end{aligned}
$$

If bidder 1 stops at $\mathrm{s}$ and if it is accepted, his ex interim payoff at $\mathrm{s}$ is:

$$
\begin{aligned}
\pi(\text { drop } ; v)= & u(v-s+\omega) \operatorname{Prob}\left\{1 \text { wins with bid } s \mid\left((s, v), \beta_{-1}\right)\right\} \\
& +u(\omega) \operatorname{Prob}\left\{1 \text { loses with bid } s \mid\left((s, v), \beta_{-1}\right)\right\} \\
= & u(\omega)+(u(v-s+\omega)-u(\omega)) \\
& \times \operatorname{Prob}\left\{1 \text { wins with bid } s \mid\left((s, v), \beta_{-1}\right)\right\} \\
= & u(\omega)+\frac{u(v-s+\omega)-u(\omega)}{\left(1-F_{2}(s)\right)(1-G(2 s))^{l}} \\
& \times\left\{\int_{s}^{1}\left(G\left(v_{2}+s\right)-G(2 s)\right)^{l} f_{2}\left(v_{2}\right) d v_{2}\right\}
\end{aligned}
$$

where we used the following derivations to evaluate the probability term

$$
\begin{aligned}
& \operatorname{Prob}\left\{1 \text { wins with bid } s \mid\left((s, v), \beta_{-1}\right)\right\} \\
& =\operatorname{Prob}\left\{v_{2}+s>w^{(1)} \mid \bar{v}_{2} \geq v_{2} \geq s, w^{(l)} \geq 2 s\right\} \\
& =\frac{\int_{s}^{\bar{v}_{2}} H_{l}\left(v_{2}+s \mid w^{(l)} \geq 2 s\right) f_{2}\left(v_{2}\right) d v_{2}}{1-F_{2}(s)} \\
& =\frac{\int_{s}^{\bar{v}_{2}}\left(G\left(v_{2}+s\right)-G(2 s)\right)^{l} f_{2}\left(v_{2}\right) d v_{2}}{\left(1-F_{2}(s)\right)(1-G(2 s))^{l}}
\end{aligned}
$$


If bidder 1 does not stop at $s$ he bids up truthfully since bidder 2 stops. Bidder 1 wins in the continuation game against bidder 3 in the event that $\left\{v+s>w^{(1)}\right\}$ and pays $w^{(1)}-s$ when he wins.

$$
\begin{aligned}
\pi(\text { continue } ;, s)= & \int_{2 s}^{v+s} u\left(v+s-w^{(1)}+\omega\right) h_{l}\left(w^{(1)} \mid w^{(l)} \geq 2 s\right) d w^{(1)} \\
& +u(\omega)\left(1-\int_{2 s}^{v+s} h_{l}\left(w^{(1)} \mid w^{(l)} \geq 2 s\right) d w^{(1)}\right) \\
= & u(\omega)+\int_{2 s}^{v+s}\left(u\left(v+s-w^{(1)}+\omega\right)-u(\omega)\right) \\
& \times h_{l}\left(w^{(1)} \mid w^{(l)} \geq 2 s\right) d w^{(1)} \\
= & u(\omega)+\int_{2 s}^{v+s}(u(v+s-t+\omega)-u(\omega)) h_{l}(t \mid t \geq 2 s) d t \\
= & u(\omega)+(u(v-s+\omega)-u(\omega))\left(-H_{l}(2 s \mid 2 s \geq 2 s)\right) \\
& +\int_{2 s}^{v+s} u^{\prime}(v+s-t+\omega) H_{l}(t \mid t \geq 2 s) d t \\
= & u(\omega)+\int_{2 s}^{v+s} u^{\prime}(v+s-t+\omega) H_{l}(t \mid t \geq 2 s) \\
& \times d t\left(\operatorname{since} H_{l}(2 s \mid 2 s \geq 2 s)=0\right)
\end{aligned}
$$

The difference (8)-(7) is:

$$
\begin{aligned}
\Delta E U(v, s):= & \pi(\text { continue } ; v, s)-\pi(\text { drop; }, s) \\
= & \frac{u(v-s+\omega)-u(\omega)}{\left(1-F_{2}(s)\right)(1-G(2 s))^{l}} \\
& \times\left\{\int_{s}^{1}\left(G\left(v_{2}+s\right)-G(2 s)\right)^{l} f_{2}\left(v_{2}\right) d v_{2}\right\} \\
& -\int_{2 s}^{v+s} u^{\prime}(v+s-t+\omega) \frac{(G(t)-G(2 s))^{l}}{(1-G(2 s))^{l}} d t \\
\operatorname{sign} \Delta E U(v, r)= & \operatorname{sign}\left\{\frac{u(v-s+\omega)-u(\omega)}{\left(1-F_{2}(s)\right)}\right. \\
& \times\left(\int_{s}^{1}\left(G\left(v_{2}+s\right)-G(2 s)\right)^{l} f_{2}\left(v_{2}\right) d v_{2}\right) \\
& \left.-\int_{s}^{v} u^{\prime}(v-y+\omega)(G(y+s)-G(2 s))^{l} d y\right\} \\
& \times(\operatorname{change} \text { variable y }=\mathrm{t}-\mathrm{s}) \\
= & \operatorname{sign}\left\{( u ( v - s + \omega ) - u ( \omega ) ) \left(\int_{s}^{1}\left(G\left(v_{2}+s\right)-G(2 s)\right)^{l}\right.\right. \\
& \times \frac{f_{2}\left(v_{2}\right)}{\left(1-F_{2}(s)\right)} d v_{2}-\int_{s}^{v} \frac{u^{\prime}(v-y+\omega)}{u(v-s+\omega)-u(\omega)}
\end{aligned}
$$




$$
\begin{aligned}
& \left.\left.\times(G(y+s)-G(2 s))^{l} d y\right)\right\} \\
= & \operatorname{sign}\left\{\int_{s}^{1}(G(t+s)-G(2 s))^{l} \frac{f_{2}(t)}{\left(1-F_{2}(s)\right)} d t\right. \\
& \left.-\int_{s}^{v} L(v, t, s, \omega)(G(t+s)-G(2 s))^{l} d t\right\}
\end{aligned}
$$

We show below (Lemma 2) that $Q(v):=\int_{s}^{v} L(v, t, s, \omega)(G(t+s)-G(2 s))^{l} d t$ is monotone increasing in $v$ and thus it is maximized at $v=1$.

Therefore we show $\triangle \mathrm{EU}(\mathrm{v}, \mathrm{s})>0 \forall v$

$$
\begin{aligned}
& \Longleftrightarrow \int_{s}^{1}(G(t+s)-G(2 s))^{l} \frac{f_{2}(t)}{\left(1-F_{2}(s)\right)} d t \\
& >\int_{s}^{1} L(1, t, s, \omega)(G(t+s)-G(2 s))^{l} d t \\
& \Longleftrightarrow \int_{s}^{1}(G(t+s)-G(2 s))^{l}\left(\frac{f_{2}(t)}{\left(1-F_{2}(s)\right)}-L(1, t, s, \omega)\right) d t>0
\end{aligned}
$$

Lemma $2 Q(v):=\int_{s}^{v} L(v, t, s, \omega)(G(t+s)-G(2 s))^{l} d t$ is monotone increasing in $v$.

Proof Lemma 2 First some claims:

\section{Claim 4}

$$
\begin{gathered}
\int_{r}^{v} \frac{u^{\prime \prime}(v-t+\omega)}{u(v-s+\omega)-u(\omega)}(G(t+s)-G(2 s))^{l} d t \\
>(G(v+s)-G(2 s))^{l} \frac{u^{\prime}(v-s+\omega)-u^{\prime}(\omega)}{u(v-s+\omega)-u(\omega)}
\end{gathered}
$$

Proof Claim 4 Similar to Claim 2

Claim $5(G(v+s)-G(2 s))^{l}>Q(v)$

Proof Claim 5 Similar to Claim 3

Now we prove the lemma by showing $Q^{\prime}(v)>0$

$$
\begin{aligned}
Q^{\prime}(v)= & L(v, v, s, \omega)(G(v+s)-G(2 s))^{l} \\
& +\int_{s}^{v} \frac{\partial L(v, t, s, \omega)}{\partial v}(G(t+s)-G(2 s))^{l} d t \\
= & L(v, v, s, \omega)(G(v+s)-G(2 s))^{l} \\
& -\int_{r}^{v} \frac{\partial L(v, t, s, \omega)}{\partial t}(G(t+s)-G(2 s))^{l} d t
\end{aligned}
$$




$$
\begin{aligned}
& -L(v, s, s, \omega) \int_{r}^{v} L(v, t, s, \omega)(G(t+s)-G(2 s))^{l} d t \quad \quad \text { (claim 1a) } \\
= & L(v, v, s, \omega)(G(v+s)-G(2 s))^{l} \\
& +\int_{r}^{v} \frac{u^{\prime \prime}(v-t+\omega)}{u(v-s+\omega)-u(\omega)}(G(t+s)-G(2 s))^{l} d t-L(v, s, s, \omega) Q(v) \\
> & L(v, v, s, \omega)(G(v+s)-G(2 s))^{l} \\
& +(G(v+s)-G(2 s))^{l} \frac{u^{\prime}(v-s+\omega)-u^{\prime}(\omega)}{u(v-s+\omega)-u(\omega)} \\
& -L(v, s, s, \omega) Q(v) \\
= & L(v, v, s, \omega)(G(v+s)-G(2 s))^{l} \\
& +(G(v+s)-G(2 s))^{l}(L(v, s, s, \omega)-L(v, v, s, \omega)) \\
& -L(v, s, s, \omega) Q(v) \\
= & L(v, s, s, \omega)\left((G(v+s)-G(2 s))^{l}-Q(v)\right)>0
\end{aligned}
$$

(claim 5 and $u(x)$ concave)

Theorem 3 If the environment $e=\left\{F_{1}, F_{2}, G, u_{1}, u_{2}, u_{3}, \omega_{1}, \omega_{2}, \omega_{3}\right\}$ admits a nonbidding equilibrium then so does the environment $\tilde{e}=\left\{F_{1}, F_{2}, G, \tilde{u}_{1}, \tilde{u}_{2}, u_{3}, \omega_{1}, \omega_{2}\right.$, $\left.\omega_{3}\right\}$ where $\tilde{u}_{i}(x)$ is such that $\tilde{A}_{i}(x)<A_{i}(x)$, for $\forall x$ and $i=1,2$, i.e., where small bidders are less risk-averse. If the environment $\tilde{e}$ does not admit non-bidding in a perfect Bayesian equilibrium, neither does the environment e.

Proof of Theorem 3 We will show that the left hand side of the non-bidding equilibrium condition (1) decreases as $A(x)$ increases. First, we describe the left hand side as a function of the utility function

$$
N B(u):=\int_{r}^{1} G(t+r)\left(\frac{f(t)}{1-F(r)}-L(u ; 1, t, r, \omega)\right) d t
$$

Let $\tilde{u}(x), u(x)$ be two utility functions and $\tilde{A}(x), A(x)$ the corresponding ArrowPratt measures such that $\tilde{A}(x)<A(x)$ for $\forall x$. We need to show

$$
N B(\tilde{u})>N B(u) \Longleftrightarrow \int_{r}^{1} G(t+r)(L(u ; 1, t, r, \omega)-L(\tilde{u} ; 1, t, r, \omega)) d t>0
$$

Define $\Delta(t):=L(u ; 1, t, r, \omega)-L(\tilde{u} ; 1, t, r, \omega)$

$\int_{r}^{1} L(u ; 1, t, r, \omega) d t=1 \forall u, t, r, \omega$, therefore

$$
\int_{r}^{1} \Delta(t) d t=0
$$

We will show that $\Delta(t)$ has always the form in Fig. 7. Precisely we will show $\Delta(1)>0$ and $\Delta(t)=0$ has exactly one root. Due to (10) the positive and the negative areas are 


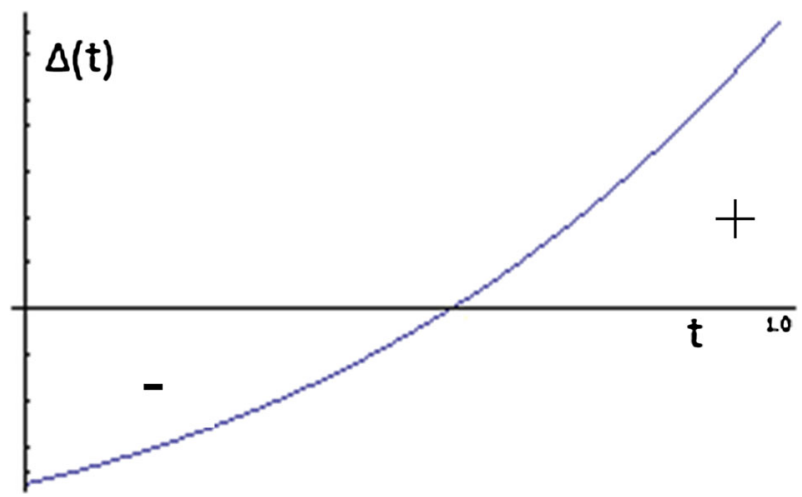

Fig. 7 Shape of $\Delta(\mathrm{t})$

equal. Since $G(x)$ is increasing, the positive area is multiplied by greater values than the negative area, hence inequality (9) is true.

To show $\Delta(1)>0$ we make use of the following lemma, proven in (Pratt 1964):

$$
\begin{aligned}
A_{2}(x)>A_{1}(x) \forall x & \Longleftrightarrow \frac{u_{2}(y)-u_{2}(x)}{u_{2}^{\prime}(w)} \\
& <\frac{u_{1}(y)-u_{1}(x)}{u_{1}^{\prime}(w)} \text { for } \forall(w, x, y): w \leq x \leq y
\end{aligned}
$$

Let $w=x=\omega, y=\omega+1-r$, then we get:

$$
\begin{aligned}
A(x)>\tilde{A}(x) \forall x & \Longleftrightarrow \frac{u(\omega+1-r)-u(\omega)}{u^{\prime}(\omega)}<\frac{\tilde{u}(\omega+1-r)-\tilde{u}(\omega)}{\tilde{u}^{\prime}(\omega)} \\
& \Longleftrightarrow \frac{u^{\prime}(\omega)}{u(\omega+1-r)-u(\omega)}>\frac{\tilde{u}^{\prime}(\omega)}{\tilde{u}(\omega+1-r)-\tilde{u}(\omega)} \\
& \Longleftrightarrow L(u ; 1, t, r, \omega)>L(\tilde{u} ; 1, t, r, \omega) \Longleftrightarrow \Delta(1)>0
\end{aligned}
$$

We proceed to show $\Delta(t)=0$ has exactly one root:

$$
\begin{aligned}
\frac{\partial L(v, t, r, \omega)}{\partial t} & =\frac{-u^{\prime \prime}(v-t+\omega)}{u(v-r+\omega)-u(\omega)}=\frac{-u^{\prime \prime}(v-t+\omega)}{u^{\prime}(v-t+\omega)} \frac{u^{\prime}(v-t+\omega)}{u(v-r+\omega)-u(\omega)} \\
& =A(v-t+\omega) L(v, t, r, \omega) \\
\frac{\partial \Delta(t)}{\partial t} & =A(1-t+\omega) L(u ; 1, t, r, \omega)-\tilde{A}(1-t+\omega) L(\tilde{u} ; 1, t, r, \omega)
\end{aligned}
$$

Suppose there are two values of $\mathrm{t}, \mathrm{t}_{2}^{*}$ and $\mathrm{t}_{1}^{*}$ with $\mathrm{t}_{2}^{*}>\mathrm{t}_{1}^{*}$ such as $\Delta\left(\mathrm{t}_{1}^{*}\right)=\Delta\left(\mathrm{t}_{2}^{*}\right)=0$.

Since $L\left(u ; 1, \mathrm{t}_{1}^{*}, r, \omega\right)=L\left(\tilde{u} ; 1, \mathrm{t}_{1}^{*}, r, \omega\right)$ and $A\left(1-\mathrm{t}_{1}^{*}+\omega\right)>\tilde{A}\left(1-\mathrm{t}_{1}^{*}+\omega\right)$ It follows $\frac{\partial \Delta\left(\mathrm{t}_{1}^{*}\right)}{\partial t}>0$. Thus:

$$
\forall t \in\left(\mathrm{t}_{1}^{*}, \mathrm{t}_{2}^{*}\right) \Delta(\mathrm{t})>0
$$


Using the mean value theorem of differential calculus, we get that $\exists \mathrm{t}^{\mathrm{c}} \in\left(\mathrm{t}_{1}^{*}, \mathrm{t}_{2}^{*}\right)$ with

$$
\begin{aligned}
\frac{\partial \Delta\left(\mathrm{t}^{\mathrm{c}}\right)}{\partial t} & =\frac{\Delta\left(\mathrm{t}_{2}^{*}\right)-\Delta\left(\mathrm{t}_{1}^{*}\right)}{\mathrm{t}_{2}^{*}-\mathrm{t}_{1}^{*}}=0 \\
& \Longleftrightarrow A\left(1-\mathrm{t}^{\mathrm{c}}+\omega\right) L\left(u ; 1, \mathrm{t}^{\mathrm{c}}, r, \omega\right)-\tilde{A}\left(1-\mathrm{t}^{\mathrm{c}}+\omega\right) L\left(\tilde{u} ; 1, \mathrm{t}^{\mathrm{c}}, r, \omega\right)=0 \\
& \Longleftrightarrow L\left(u ; 1, \mathrm{t}^{\mathrm{c}}, r, \omega\right)<L\left(\tilde{u} ; 1, \mathrm{t}^{\mathrm{c}}, r, \omega\right) \quad\left(\text { since } A\left(1-\mathrm{t}^{\mathrm{c}}+\omega\right)\right. \\
& \left.>\tilde{A}\left(1-\mathrm{t}^{\mathrm{c}}+\omega\right)\right) \\
& \Longleftrightarrow \Delta\left(\mathrm{t}^{\mathrm{c}}\right)<0 \text { which is a contradiction to }(11) .
\end{aligned}
$$

Thus there cannot exist two (or more) values of $t, t_{1}^{*}$ and $t_{2}^{*}$ with $t_{2}^{*}>t_{1}^{*}$ such as $\Delta\left(\mathrm{t}_{1}^{*}\right)=\Delta\left(\mathrm{t}_{2}^{*}\right)=0$. Due to (10) and (11) there is at least one root. Thus there is exactly one root. The last step of the proof is to formally show that the positive area is multiplied by greater values than the negative area, which implies that the integral in (9) is positive.

Define $t^{*}$ as $\Delta\left(t^{*}\right)=0$. Then,

$$
-\int_{r}^{t^{*}} G(t+r) \Delta(t) d t<-G\left(t^{*}+r\right) \int_{r}^{t^{*}} \Delta(t) d t<\int_{t^{*}}^{1} G(t+r) \Delta(t) d t
$$

(since for any $t$ in $\left[r, t^{*}\right] G(t+r)<G\left(t^{*}+r\right)$ and $G$ increasing)

$$
\begin{aligned}
\Longleftrightarrow & \int_{r}^{t^{*}} G(t+r) \Delta(t) d t+\int_{t^{*}}^{1} G(t+r) \Delta(t) d t>0 \\
\Longleftrightarrow & \int_{r}^{1} G(t+r) \Delta(t) d t>0 \Longleftrightarrow \int_{r}^{1} G(t+r)(L(u ; 1, t, r, \omega) \\
& \quad-L(\tilde{u} ; 1, t, r, \omega)) d t>0
\end{aligned}
$$

Corollary 6 Suppose $u_{1}$ is a CRRA or DARA utility function. If the environment $e=\left\{F_{1}, F_{2}, F_{3}, u_{1}, u_{2}, u_{3}, \omega_{1}, \omega_{2}, \omega_{3}\right\}$ admits a non-bidding equilibrium then so does the environment $\tilde{e}=\left\{F_{1}, F_{2}, F_{3}, u_{1}, u_{2}, u_{3}, \tilde{\omega}_{1}, \omega_{2}, \omega_{3}\right\}$ where $\tilde{\omega}_{1}>\omega_{1}$.

Proof of Corollary 6 First, we express the left hand side as a function of $\omega$ as

$$
N B(\omega):=\int_{r}^{1} G(t+r)\left(\frac{f(t)}{1-F(r)}-L(u ; 1, t, r, \omega)\right) d t
$$

We need to show $N B(\omega+\delta)>N B(\omega) \Longleftrightarrow \int_{r}^{1} G(t+r)(L(u ; 1, t, r, \omega)-$ $L(u ; 1, t, r, \omega+\delta)) d t>0$

Define $\Delta(t, \delta):=L(u ; 1, t, r, \omega)-L(u ; 1, t, r, \omega+\delta)$

We first show $\Delta(1, \delta)>0 \forall \delta>0$ : 
Integrating $A(\omega+\delta)<A(\omega)$ from $x$ to $y$ we get

$$
\begin{aligned}
\int_{x}^{y} \frac{u^{\prime \prime}(\omega+\delta)}{u^{\prime}(\omega+\delta)} d \omega & >\int_{x}^{y} \frac{u^{\prime \prime}(\omega)}{u^{\prime}(\omega)} d \omega \log \left(\frac{u^{\prime}(y+\delta)}{u^{\prime}(x+\delta)}\right)>\log \left(\frac{u^{\prime}(y)}{u^{\prime}(x)}\right) \\
& \Longleftrightarrow \frac{u^{\prime}(y+\delta)}{u^{\prime}(x+\delta)}>\frac{u^{\prime}(y)}{u^{\prime}(x)} \text { for } x<y, \delta>0 \\
q(y) & :=\frac{u(y)-u(x)}{u^{\prime}(x)}-\frac{u(y+\delta)-u(x+\delta)}{u^{\prime}(x+\delta)} \\
q(y) & =\frac{u^{\prime}(y)}{u^{\prime}(x)}-\frac{u^{\prime}(y+\delta)}{u^{\prime}(x+\delta)}<0 \text { due to the last inequality }
\end{aligned}
$$

Apply the mean value theorem on $[x, y]$ :

$$
\frac{q(y)-q(x)}{y-x}<0 \Longleftrightarrow q(y)<0 \text { since } q(x)=0, y-x>0
$$

Now replace in $q(y)<0 \quad y$ with $\omega+1-r$ and $x$ with $\omega$ :

$$
\begin{gathered}
\frac{u(\omega+1-r)-u(\omega)}{u^{\prime}(\omega)}-\frac{u(\omega+1-r+\delta)-u(\omega+\delta)}{u^{\prime}(\omega+\delta)}<0 \\
\Longleftrightarrow L(1,1, r, \omega+\delta)<L(1,1, r, \omega) \Longleftrightarrow \Delta(1, \delta)>0
\end{gathered}
$$

We proceed to show $\Delta(t)=0$ has exactly one root:

$$
\begin{aligned}
\frac{\partial L(v, t, r, \omega)}{\partial t} & =\frac{-u^{\prime \prime}(v-t+\omega)}{u(v-r+\omega)-u(\omega)}=\frac{-u^{\prime \prime}(v-t+\omega)}{u^{\prime}(v-t+\omega)} \frac{u^{\prime}(v-t+\omega)}{u(v-r+\omega)-u(\omega)} \\
& =A(v-t+\omega) L(v, t, r, \omega) \\
\frac{\partial \Delta(t)}{\partial t} & =A(1-t+\omega) L(u ; 1, t, r, \omega)-\tilde{A}(1-t+\omega+\delta) L(u ; 1, t, r, \omega+\delta)
\end{aligned}
$$

Suppose there are two values of $\mathrm{t}, \mathrm{t}_{2}^{*}$ and $\mathrm{t}_{1}^{*}$ with $\mathrm{t}_{2}^{*}>\mathrm{t}_{1}^{*}$ such as $\Delta\left(\mathrm{t}_{1}^{*}\right)=\Delta\left(\mathrm{t}_{2}^{*}\right)=0$.

Since $L\left(u ; 1, \mathrm{t}_{1}^{*}, r, \omega\right)=L\left(u ; 1, \mathrm{t}_{1}^{*}, r, \omega+\delta\right)$ and $A\left(1-\mathrm{t}_{1}^{*}+\omega\right)>A\left(1-\mathrm{t}_{1}^{*}+\right.$ $\omega+\delta)$

It follows $\frac{\partial \Delta\left(\mathrm{t}_{1}^{*}\right)}{\partial t}>0$. Thus:

$$
\forall t \in\left(\mathrm{t}_{1}^{*}, \mathrm{t}_{2}^{*}\right) \Delta(\mathrm{t})>0
$$

Using the mean value theorem of differential calculus, we get that $\exists \mathrm{t}^{\mathrm{c}} \in\left(\mathrm{t}_{1}^{*}, \mathrm{t}_{2}^{*}\right)$ with

$$
\begin{aligned}
\frac{\partial \Delta\left(\mathrm{t}^{\mathrm{c}}\right)}{\partial t}= & \frac{\Delta\left(\mathrm{t}_{2}^{*}\right)-\Delta\left(\mathrm{t}_{1}^{*}\right)}{\mathrm{t}_{2}^{*}-\mathrm{t}_{1}^{*}}=0 \\
\Longleftrightarrow & A\left(1-\mathrm{t}^{\mathrm{c}}+\omega\right) L\left(u ; 1, \mathrm{t}^{\mathrm{c}}, r, \omega\right) \\
& \quad-A\left(1-\mathrm{t}^{\mathrm{c}}+\omega+\delta\right) L\left(u ; 1, \mathrm{t}^{\mathrm{c}}, r, \omega+\delta\right)=0
\end{aligned}
$$




$$
\begin{aligned}
& \Longleftrightarrow L\left(u ; 1, \mathrm{t}^{\mathrm{c}}, r, \omega\right)<L\left(u ; 1, \mathrm{t}^{\mathrm{c}}, r, \omega+\delta\right) \quad\left(\text { since } A\left(1-\mathrm{t}^{\mathrm{c}}+\omega\right)\right. \\
& \left.>A\left(1-\mathrm{t}^{\mathrm{c}}+\omega+\delta\right)\right) \\
& \Longleftrightarrow \Delta\left(\mathrm{t}^{\mathrm{c}}\right)<0 \text { which is a contradiction to (12) }
\end{aligned}
$$

Theorem 4 Suppose $=0$. If the environmente $=\left\{F_{1}, F_{2}, G, u_{1}, u_{2}, u_{3}, \omega_{1}, \omega_{2}, \omega_{3}\right\}$ admits a non-bidding equilibrium then so does the environment $\tilde{e}=\left\{\tilde{F}_{1}, F_{2}, G, u_{1}\right.$, $\left.u_{2}, u_{3}, \omega_{1}, \omega_{2}, \omega_{3}\right\}$ where $\tilde{F}_{1} \succcurlyeq_{F S D} F_{1}$.

Proof of Theorem 4:

$$
\begin{aligned}
N B\left(\tilde{F}_{1}\right)>N B\left(F_{1}\right) \Longleftrightarrow & \int_{0}^{1} G(t)\left(\tilde{f}_{1}(t)-f_{1}(t)\right) d t \geq 0 \\
\Longleftrightarrow & G(1)\left(\tilde{F}_{1}(1)-F_{1}(1)\right) \\
& -\int_{0}^{1} g(t)\left(\tilde{F}_{1}(t)-F_{1}(t)\right) d t \geq 0 \\
& \Longleftrightarrow \int_{0}^{1} g(t)\left(\tilde{F}_{1}(t)-F_{1}(t)\right) d t \leq 0
\end{aligned}
$$

The last inequality holds since $g(t)>0$ and $\tilde{F}_{1} \succcurlyeq_{F S D} F_{1}$.

Theorem 5 Suppose additionally $g$ is non-increasing. Then the weaker condition $\tilde{F}_{1} \succcurlyeq_{S S D} F_{1}$ is sufficient for Theorem 4 to hold.

Proof of Theorem 5 If $g(t)$ is non-increasing, then only the weaker second-order stochastic dominance is required:

$$
\Delta(t):=F_{1}(t)-\tilde{F}_{1}(t) .
$$

Since $\Delta(0+\varepsilon) \geq 0$ (for a small $\varepsilon$-due to SSD), if $\Delta$ has up to one roots, (13) holds immediately. Suppose now $\Delta$ has $n+1$ roots $t_{0}<\cdots<t_{n}$. $\tilde{F}_{1} \succcurlyeq S S D F_{1} \Rightarrow$ $\int_{t_{0}}^{t_{i}} \Delta(t) d t \geq 0 \forall i$.

$\Delta(t) \geq 0 \forall t \in\left[t_{i-1}, t_{i}\right]$, if $i$ odd and $\Delta(t) \leq 0 \forall t \in\left[t_{i}, t_{i+1}\right]$ if $i$ even.

Let $I_{i}:=\int_{t_{i-1}}^{t_{i}} g(t) \Delta(t) d t$. Since g non-increasing, $I_{2 k-1} \geq g\left(t_{2 k-1}\right) \int_{t_{2 k-2}}^{t_{2 k-1}}$ $\Delta(t) d t \geq 0$ and

$$
I_{2 k} \geq g\left(t_{2 k-1}\right) \int_{t_{2 k-1}}^{t_{2 k}} \Delta(t) d t \quad \forall k \in \mathbb{N}
$$

It can be observed that the sign of $\Delta(\mathrm{t})$ is positive in $\left[\mathrm{t}_{\mathrm{i}-1}, \mathrm{t}_{\mathrm{i}}\right]$, if $i$ is odd, else negative. The inequalities concerning the integrals $I_{i}$ hold since $g$ is non-increasing (see Fig. 8)

Now let $S_{n}:=\sum_{i=1}^{n} I_{i}$. We will show by induction that $S_{n} \geq 0 \forall n$ [this immediately implies (13)]. 


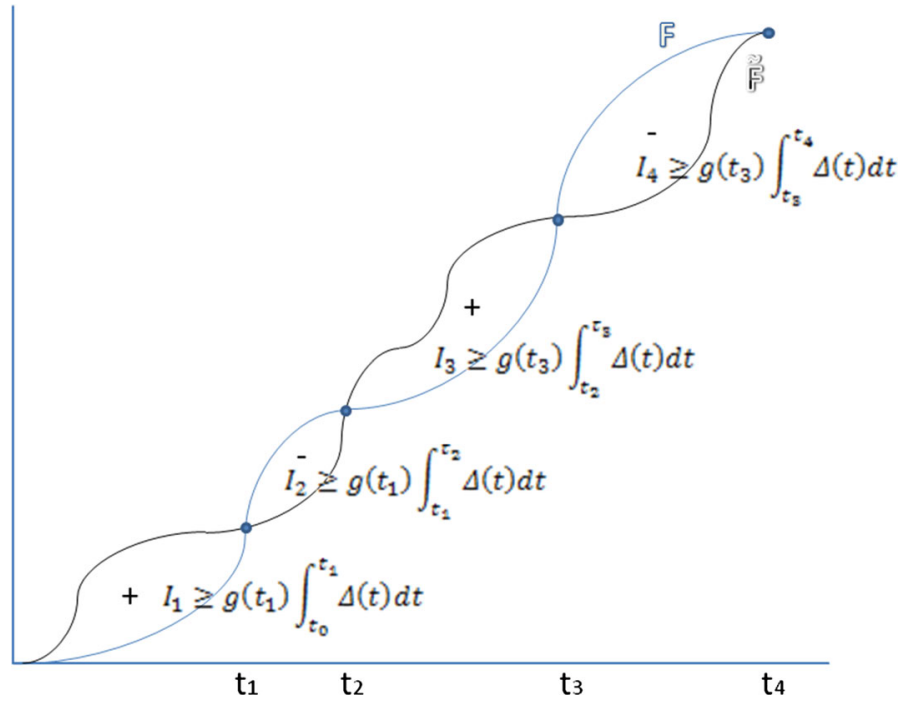

Fig. 8 Second order stochastic dominance $\tilde{\mathbf{F}} \succcurlyeq_{\text {SSD }}$ but not $\tilde{\mathbf{F}} \succcurlyeq_{\text {FSD }} \mathbf{F}$

$$
\begin{aligned}
& S_{1} \geq 0 \text { since } S_{1}=I_{1} \geq g\left(t_{1}\right) \int_{t_{0}}^{t_{1}} \Delta(t) d t \geq 0 \\
& S_{2}=I_{1}+I_{2} \geq g\left(t_{1}\right) \int_{t_{0}}^{t_{2}} \Delta(t) d t \geq 0 \text { due to } \tilde{F}_{1} \succcurlyeq_{F S D} F_{1} .
\end{aligned}
$$

We assume $S_{2 k} \geq g\left(t_{2 k-1}\right) \int_{t_{0}}^{t_{2 k}} \Delta(t) d t \geq 0$ and show $S_{2 k+2} \geq g\left(t_{2 k+1}\right) \int_{t_{0}}^{t_{2 k+2}}$ $\Delta(t) d t \geq 0$

$$
\begin{aligned}
S_{2 k+2}= & S_{2 k}+I_{2 k+1}+I_{2 k+2} \geq S_{2 k}+g\left(t_{2 k+1}\right) \\
& \times \int_{t_{2 k}}^{t_{2 k+2}} \Delta(t) d t \geq g\left(t_{2 k-1}\right) \int_{t_{0}}^{t_{2 k}} \Delta(t) d t \\
& +g\left(t_{2 k+1}\right) \int_{t_{2 k}}^{t_{2 k+2}} \Delta(t) d t \geq g\left(t_{2 k+1}\right) \\
& \times \int_{t_{0}}^{t_{2 k}} \Delta(t) d t+g\left(t_{2 k+1}\right) \int_{t_{2 k}}^{t_{2 k+2}} \Delta(t) d t \\
= & g\left(t_{2 k+1}\right) \int_{t_{0}}^{t_{2 k+2}} \Delta(t) d t \geq 0
\end{aligned}
$$

In addition $S_{2 k+1}=S_{2 k}+I_{2 k+1} \geq 0$

\section{References}

Airiau S, Sen S (2003) Strategic bidding for multiple units in simultaneous and sequential auctions. Group Decisi Negot 12(5):397-413 
Arrow KJ (1965) Aspects of the theory of risk-bearing. Yrjö Jahnssonin Säätiö

Athey S (2001) Single crossing properties and the existence of pure strategy equilibria in games of incomplete information. Econometrica 69(4):861-889

Ausubel LM, Milgrom P (2006a) Ascending proxy auctions. Comb Auctions 79-98

Ausubel LM, Milgrom P (2006b) The lovely but lonely vickrey auction. Comb Auctions 17-40

Ausubel LM, Milgrom PR (2002) Ascending auctions with package bidding. BE J Theor Econ 1(1):1

Banks J, Olson M, Porter D, Rassenti S, Smith V (2003) Theory, experiment and the federal communications commission spectrum auctions. J Econ Behav Organ 51(3):303-350

Bichler M, Shabalin P, Wolf J (2013) Do core-selecting combinatorial clock auctions always lead to high efficiency? An experimental analysis of spectrum auction designs. Exp Econ 16(4):511-545

Bikhchandani S, Ostroy JM (2002) The package assignment model. J Econ Theory 107(2):377-406

Chen K-Y, Plott CR (1998) Nonlinear behavior in sealed bid first price auctions. Games Econ Behav 25(1):34-78

Clarke EH (1971) Multipart pricing of public goods. Public Choice 11(1):17-33

Cox JC, Smith VL, Walker JM (1988) Theory and individual behavior of first-price auctions. J Risk Uncertain 1(1):61-99

Cramton P (2013) Spectrum auction design. Rev Ind Organ 42(2):161-190

Cramton P, Day R (2012) The quadratic core-selecting payment rule for combinatorial auctions. Oper Res 60(3):588-603

Cramton P, Shoham Y, Steinberg R (2006) Combinatorial auctions

Day RW, Raghavan S (2007) Fair payments for efficient allocations in public sector combinatorial auctions. Manag Sci 53(9):1389-1406

Drexl A, Jørnsten K, Knof D (2009) Non-linear anonymous pricing combinatorial auctions. Eur J Oper Res 199(1):296-302

Fibich G, Gavious A (2010) Large auctions with risk-averse bidders. Int J Game Theory 39(3):359-390

Fibich G, Gavious A, Sela A (2006) All-pay auctions with risk-averse players. Int J Game Theory 34(4):583599

Goeree J, Lien Y (2009) An equilibrium analysis of the simultaneous ascending auction

Goeree JK, Holt CA (2010) Hierarchical package bidding: a paper \& pencil combinatorial auction. Games Econ Behav 70(1):146-169

Goeree JK, Lien Y (2013) On the impossibility of core-selecting auctions. Theor Econ

Green J, Laffont JJ (1977) Characterization of satisfactory mechanisms for the revelation of preferences for public goods. Econ J Econ Soc 427-438

Groves T (1973) Incentives in teams. Econ J Econ Soc 617-631

Kirchkamp O, Reiss JP (2011) Out-of equilibrium bids in auctions: wrong expectations or wrong bids. Econ J 121(557):1361-1397

Kwasnica AM, Ledyard JO, Porter D, DeMartini C (2005) A new and improved design for multiobject iterative auctions. Manag Sci 51(3):419-434

Lehmann D, Müller R, Sandholm T (2006) The winner determination problem. Comb Auctions 297-317

Parkes DC, Ungar LH (2000) Iterative combinatorial auctions: theory and practice. MIT Press, Cambridge

Porter D, Rassenti S, Roopnarine A, Smith V (2003) Combinatorial auction design. Proc Natl Acad Sci USA 100(19):11153

Pratt JW (1964) Risk aversion in the small and in the large. Econ J Econ Soc 122-136

Riley JG, Samuelson WF (1981) Optimal auctions. Am Econ Rev 71(3):381-392

Rothkopf MH, Pekeč A, Harstad RM (1998) Computationally manageable combinational auctions. Manag Sci 44(8):1131-1147

Sano R (2011) Non-bidding equilibrium in an ascending core-selecting auction. Games Econ Behav

Scheffel T, Pikovsky A, Bichler M, Guler K (2011) An experimental comparison of linear and nonlinear price combinatorial auctions. Inf Syst Res 22(2):346-368

Schneider S, Shabalin P, Bichler M (2010) On the robustness of non-linear personalized price combinatorial auctions. Eur J Oper Res 206(1):248-259

Vickrey W (1961) Counterspeculation, auctions, and competitive sealed tenders. J Finance 16(1):8-37

Xia M, Koehler GJ, Whinston AB (2004) Pricing combinatorial auctions. Eur J Oper Res 154(1):251-270 